\title{
SPHERICAL INTERFACE DYNAMOS: MATHEMATICAL THEORY, FINITE ELEMENT APPROXIMATION, AND APPLICATION*
}

\author{
KIT HUNG $\mathrm{CHAN}^{\dagger}, \mathrm{KEKE} \mathrm{ZHANG}{ }^{\dagger}$, AND JUN ZOU
}

\begin{abstract}
Stellar magnetic activities such as the 11-year sunspot cycle are the manifestation of magnetohydrodynamic dynamo processes taking place in the deep interiors of stars. This paper is concerned with the mathematical theory and finite element approximation of mean-field spherical dynamos and their astrophysical application. We first investigate the existence, uniqueness, and stability of the dynamo system governed by a set of nonlinear PDEs with discontinuous physical coefficients in spherical geometry, and characterize the system by a saddle-point type variational form. Then we propose a fully discrete finite element approximation to the dynamo system and study its convergence and stability. For the astrophysical application, we perform some fully threedimensional numerical simulations of a solar interface dynamo using the proposed algorithm, which successfully generates the equatorially propagating dynamo wave with a period of about 11 years similar to that of the Sun.
\end{abstract}

Key words. spherical interface dynamo, well-posedness, finite element analysis

AMS subject classifications. 65M60, 65M12,65M15

DOI. $10.1137 / 050635596$

1. Introduction. Many astrophysical bodies possess intrinsic magnetic fields. The radio signals in connection with Jupiter's magnetic field were first observed more than a half century ago [8] and Jupiter's magnetic field was later measured by the Pioneer spacecraft [1]; the Sun's magnetic field has been observed for a long time [31] and undergone nearly periodic variations with a period of about 11 years. It has been widely accepted that large-scale planetary and stellar magnetic activities represent the manifestation of magnetohydrodynamic dynamo processes taking place in the deep interiors of planets and stars $[23,34,36,4]$. Though significant progress has been made toward the understanding of quantitative features of stellar magnetic activities, more realistic dynamo simulations in the parameter regime pertaining to stars and planets remain a tough challenge.

Nearly all current stellar and planetary numerical dynamo models employ spectral methods with spherical harmonic functions $[35,19,22,7]$. The slow Legendre transform and its global nature are computationally inefficient and severely limit the application of spectral methods to general dynamo models, especially to the models with variable physical parameters of space and time. It is becoming increasingly clear that, in order to simulate astrophysical and planetary dynamos using more realistic physical parameters [36], developing other numerically more efficient methods is necessary. The first attempt using finite element methods for numerical dynamo

* Received by the editors July 9, 2005; accepted for publication (in revised form) March 31, 2006; published electronically September 29, 2006.

http://www.siam.org/journals/sinum/44-5/63559.html

${ }^{\dagger}$ School of Mathematical Sciences, University of Exeter, Exeter, UK (k.h.chan@exeter.ac.uk, k.zhang@exeter.ac.uk). The work of the second author was supported by UK PPARC, Leverhulme and NERC grants.

${ }^{\ddagger}$ Department of Mathematics, The Chinese University of Hong Kong, Shatin, Hong Kong (zou@ math.cuhk.edu.hk). The work of this author was fully supported by Hong Kong RGC grants (Project 403403 and Project 4048/02P). 
simulations was made in [11] and proved to be very promising. The current work presents the first mathematical theory and numerical analysis for mean-field spherical dynamos and their application to astrophysical and planetary problems.

Many stars and planets like the Sun and Jupiter are convectively unstable, which drive small-scale turbulent flows as well as large-scale global circulations in their interiors. The small-scale turbulent convective flows are capable of generating largescale magnetic fields by the complex dynamo processes [24, 9]. A widely accepted theory for the generation of large-scale magnetic fields through the effect of smallscale turbulence in a conducting fluid is called the mean-field dynamo theory [23], in which a key quantity is the turbulent electromotive force defined as

$$
\mathcal{E}=<\hat{\mathbf{u}} \times \hat{\mathbf{B}}>\approx \alpha \mathbf{B},
$$

where $\langle.>$ indicates an average in the dynamo domain, $\mathbf{B}$ is the large-scale mean field, $\hat{\mathbf{u}}$ and $\hat{\mathbf{B}}$ denote the fluctuating small-scale velocity and magnetic fields, and $\alpha$ is typically a tensor describing how the small-scale flows generate the large-scale mean field. Furthermore, the small-scale dynamo simulations suggest that the turbulent electromotive force obeys the following relation [9]:

$$
\mathcal{E}=\frac{\alpha_{0} \mathbf{B}}{1+\left(\hat{R}_{m}\right)^{n}|\mathbf{B}|^{2} / B_{e q}^{2}},
$$

where $\alpha_{0}$ is constant, $0 \leq n \leq 2$ and $B_{e q}$ is the stellar equipartition field and $\hat{R}_{m}$ is the magnetic Reynolds number measuring the magnitude of the small-scale flow. The factor $\left(1+\left(\hat{R}_{m}\right)^{n}\left|\mathbf{B} / B_{e q}\right|^{2}\right)$ represents the nonlinear process of alpha quenching (the catastrophic quenching) which saturates the growing magnetic field. It should be noted that the $\hat{R}_{m}$-dependent quenching expression should be regarded as a simplified steady state expression for the nonlinear dynamo [4]. On the basis of the quenching relation (1.2), one can investigate the dynamo process of large-scale stellar magnetic fields without being complicated by the dynamic effect such as Lorentz forces. In consequence, (1.2) has been frequently used in the numerical study of astrophysical dynamos [26].

In the present study, we consider a general nonlinear kinematic dynamo for stars and planets consisting of three major zones in spherical geometry; see Figure 1. An inner radiative sphere $\Omega_{1}$ of radius $r_{1}$, with magnetic diffusivity $\lambda_{1}(\mathbf{x})$, rotates uniformly. Magnetic field $\mathbf{B}_{1}$ cannot be generated in the radiative region by dynamo action. On the top of the radiative core, there exists a turbulent convection zone $\Omega_{2}$, $r_{1} \leq r \leq r_{2}$, in which thermal instabilities drive global circulations $\mathbf{u}$ and small-scale turbulent flows $\hat{\mathbf{u}}$. Note that the effect of the small-scale turbulence in the convection zone is described by $\alpha$. In the current mean-field dynamo model, we shall use a conventional quenching formula by ignoring the $\hat{R}_{m}$-dependence in the quenching expression. The magnetic diffusivity in the convection zone is denoted by $\lambda_{2}$ while the nonlinear alpha quenching is assumed to be of the form

$$
\alpha=\frac{\alpha_{0} f(\mathbf{x}, t)}{1+\sigma\left|\mathbf{B} / B_{e q}\right|^{2}},
$$

where $f(\mathbf{x}, t)$ is a model-oriented function, $\alpha_{0}$ and $\sigma$ are constant parameters, and $\mathbf{B}$ is the generated large-scale magnetic field in the convection zone. The outer region $\Omega_{3}, r_{2} \leq r \leq r_{3}$, exterior to the convection zone is assumed to be nearly electrically 


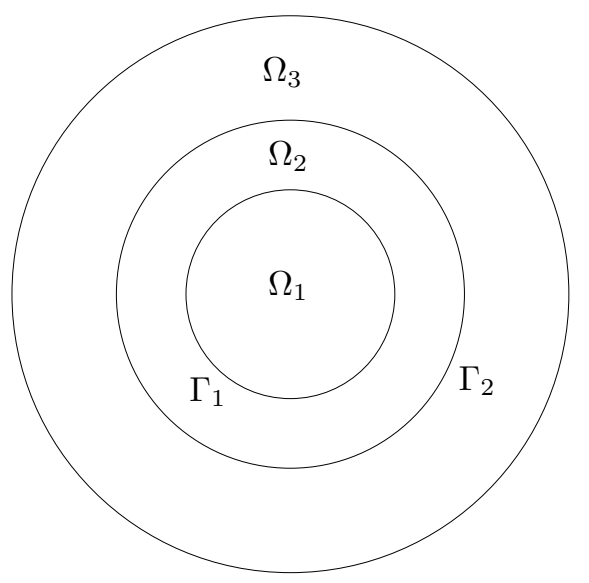

FIG. 1. Domain $\Omega$, with its inner core $\Omega_{1}$, convection zone $\Omega_{2}$, and exterior region $\Omega_{3}$.

insulating. We nondimensionalize length by the thickness of the convection zone $d=\left(r_{2}-r_{1}\right)$, the magnetic field by the equipartition field $B_{e q}$, and time by the magnetic diffusion time $d^{2} / \lambda_{2}$ of the convection zone. This leads to the three sets of dimensionless equations for the three zones in a magnetic star. For the convection fluid shell zone, we have

$$
\begin{aligned}
\frac{\partial \mathbf{B}_{2}}{\partial t}+\nabla \times\left(\nabla \times \mathbf{B}_{2}\right)= & R_{\alpha} \nabla \times\left(\frac{f(\mathbf{x}, t)}{1+\sigma\left|\mathbf{B}_{2}\right|^{2}} \mathbf{B}_{2}\right) \\
& +R_{m} \nabla \times\left(\mathbf{u} \times \mathbf{B}_{2}\right) \text { in } \Omega_{2} \times(0, T) \\
\nabla \cdot \mathbf{B}_{2}= & 0 \quad \text { in } \quad \Omega_{2} \times(0, T),
\end{aligned}
$$

where $R_{\alpha}$ is a dynamo parameter in connection with the generation process of smallscale turbulence $\hat{\mathbf{u}}$ and $R_{m}$ is the magnetic Reynolds number associated with the global circulation. For dynamo action to occur, either $R_{\alpha}$ or $R_{m}$ must be sufficiently large. The diffusion of the magnetic field $\mathbf{B}_{1}$ in the inner radiative core with a magnetic diffusivity $\beta_{1}$ can be described by

$$
\begin{aligned}
& \frac{\partial \mathbf{B}_{1}}{\partial t}+\nabla \times\left(\beta_{1}(\mathbf{x}) \nabla \times \mathbf{B}_{1}\right)=0 \quad \text { in } \quad \Omega_{1} \times(0, T), \\
& \nabla \cdot \mathbf{B}_{1}=0 \quad \text { in } \quad \Omega_{1} \times(0, T) .
\end{aligned}
$$

The outer exterior region is usually nearly electrically insulating and governed by

$$
\begin{array}{r}
\frac{\partial \mathbf{B}_{3}}{\partial t}+\nabla \times\left(\beta_{3}(\mathbf{x}) \nabla \times \mathbf{B}_{3}\right)=0 \quad \text { in } \quad \Omega_{3} \times(0, T), \\
\nabla \cdot \mathbf{B}_{3}=0 \quad \text { in } \quad \Omega_{3} \times(0, T),
\end{array}
$$

where $\beta_{3}(\mathbf{x})$ is the magnetic diffusivity of the zone.

The above model system will be complemented with the initial conditions

$$
\mathbf{B}(\mathbf{x}, 0)=\mathbf{B}_{0}(\mathbf{x}) \quad \text { in } \Omega
$$


and the boundary conditions

$$
\left(\beta_{3}(\mathbf{x}) \nabla \times \mathbf{B}_{3}\right) \times \mathbf{n}=0, \quad \mathbf{B}_{3} \cdot \mathbf{n}=0 \quad \text { on } \quad \partial \Omega \times(0, T),
$$

here and in what follows, $\mathbf{n}$ stands for the unit outward normal to the boundary $\partial \Omega$ of the entire physical domain $\Omega$, which consists of the inner core $\Omega_{1}$, the convection zone $\Omega_{2}$, and the outer exterior region $\Omega_{3}$. It should be mentioned that the shear near the solar surface, the effect of which is neglected in our interface solar dynamo model in section 6, may play an important role [3].

We shall use $\Gamma_{1}$ and $\Gamma_{2}$ to denote, respectively, the interface between the inner core and outer convection zone and between the convection zone and the outer exterior; see Figure 1. Since the magnetic diffusivity $\beta(\mathbf{x})$ has jumps across the interfaces $\Gamma_{1}$ and $\Gamma_{2}$ the magnetic field must fulfill some physical interface conditions. We shall take the following standard physical jump conditions adopted in the geodynamo modelling across the interfaces:

$$
[(\beta(\mathbf{x}) \nabla \times \mathbf{B}) \times \mathbf{n}]=0, \quad[\mathbf{B}]=0 \quad \text { on } \quad\left(\Gamma_{1} \cup \Gamma_{2}\right) \times(0, T),
$$

here and in what follows we use $[\mathbf{A}]$ to denote the quantity of jumps of $\mathbf{A}$ across the interfaces, and $\mathbf{n}$ is the outward normal of $\partial \Omega_{2}$.

Physically speaking, function $f(\mathbf{x}, t)$ and the convective flow $\mathbf{u}$ in (1.4) appear only in the fluid shell region. We shall assume that the velocity $\mathbf{u}$ is nonslip on the boundaries of the fluid shell, i.e., both $f(\mathbf{x}, t)$ and $\mathbf{u}$ vanish on $\Gamma_{1}$ and $\Gamma_{2}$. Then by viewing $f(\mathbf{x}, t)$ and $\mathbf{u}$ to be extended by zero onto the whole physical domain $\Omega$, we can unify (1.4)-(1.5), (1.6)-(1.7), and (1.8)-(1.9) in three regions $\Omega_{1}, \Omega_{2}$, and $\Omega_{3}$ as the following mean-field dynamo system:

$$
\begin{aligned}
\frac{\partial \mathbf{B}}{\partial t}+\nabla \times(\beta(\mathbf{x}) \nabla \times \mathbf{B})= & R_{\alpha} \nabla \times\left(\frac{f(\mathbf{x}, t)}{1+\sigma|\mathbf{B}|^{2}} \mathbf{B}\right) \\
& +R_{m} \nabla \times(\mathbf{u} \times \mathbf{B}) \text { in } \Omega \times(0, T) \\
\nabla \cdot \mathbf{B}= & 0 \quad \text { in } \Omega \times(0, T),
\end{aligned}
$$

where $\beta(\mathbf{x})$ represents the magnetic diffusivity $\beta_{1}(\mathbf{x}), \beta_{2}(\mathbf{x})$, and $\beta_{3}(\mathbf{x})$ in $\Omega_{1}, \Omega_{2}$, and $\Omega_{3}$,respectively, with $\beta_{2}(\mathbf{x})$ normalized to be 1 , so $\beta(\mathbf{x})$ is piecewise smooth and may have large jumps across the interfaces.

The rest of this paper is arranged as follows. Section 2 addresses the wellposedness of the mean-field dynamo system, which is then characterized in terms of a saddle-point type formulation in section 3 for the convenient approximation by finite element methods. The existing convergence theory on saddle-point systems is first generalized in section 4, and a fully discrete finite element method is then proposed and the stability and unique existence are studied. The convergence of the fully discrete scheme is established in section 5, for which the key steps are the introduction of a discrete projection operator and a modification of the Scott-Zhang operator as well as the derivations of their approximation error estimates for piecewise smooth functions. The application of the proposed numerical method to a solar interface dynamo is carried out in section 6 . Finally some concluding remarks are given in section 7 to summarize the main contributions of the paper.

2. Well-posedness of the mean-field dynamo system. In this section, we shall investigate the existence, uniqueness, and stability of the solutions to the meanfield dynamo system (1.13)-(1.14) with the initial-boundary conditions (1.10)-(1.11) 
and the interface conditions (1.12). Due to space limitations, some proof details may be omitted from time to time throughout the paper but can be found in [10].

2.1. Preliminaries. The most frequently used spaces in the subsequent analysis are the following two Sobolev spaces:

$$
\begin{aligned}
H(\operatorname{curl} ; \Omega) & =\left\{\mathbf{A} \in L^{2}(\Omega)^{3} ; \quad \operatorname{curl} \mathbf{A} \in L^{2}(\Omega)^{3}\right\}, \\
H(\operatorname{div} ; \Omega) & =\left\{\mathbf{A} \in L^{2}(\Omega)^{3} ; \quad \operatorname{div} \mathbf{A} \in L^{2}(\Omega)\right\},
\end{aligned}
$$

as well as their subspaces

$$
\begin{aligned}
H_{0}(\operatorname{curl} ; \Omega) & =\left\{\mathbf{A} \in L^{2}(\Omega)^{3} ; \quad \operatorname{curl} \mathbf{A} \in L^{2}(\Omega)^{3}, \mathbf{A} \times \mathbf{n}=0 \text { on } \partial \Omega\right\}, \\
H_{0}(\operatorname{div} ; \Omega) & =\left\{\mathbf{A} \in L^{2}(\Omega)^{3}, \quad \operatorname{div} \mathbf{A} \in L^{2}(\Omega), \mathbf{A} \cdot \mathbf{n}=0 \text { on } \partial \Omega\right\},
\end{aligned}
$$

equipped with the norms

$$
\|\mathbf{A}\|_{H(\mathbf{c u r l} ; \Omega)}=\left\{\|\mathbf{A}\|^{2}+\|\nabla \times \mathbf{A}\|^{2}\right\}^{\frac{1}{2}} ; \quad\|\mathbf{A}\|_{H(\operatorname{div} ; \Omega)}=\left\{\|\mathbf{A}\|^{2}+\|\nabla \cdot \mathbf{A}\|^{2}\right\}^{\frac{1}{2}} .
$$

In the case that the magnetic field is continuous across the interfaces, the intersection of the spaces $H(\operatorname{curl} ; \Omega)$ and $H(\operatorname{div} ; \Omega)$ is the natural Sobolev space to be adopted:

$$
\begin{gathered}
H(\operatorname{curl}, \operatorname{div} ; \Omega)=\left\{\mathbf{A} \in L^{2}(\Omega)^{3} ; \quad \operatorname{curl} \mathbf{A} \in L^{2}(\Omega)^{3}, \operatorname{div} \mathbf{A} \in L^{2}(\Omega)\right\}, \\
H_{0}(\mathbf{c u r l}, \operatorname{div} ; \Omega)=\{\mathbf{A} \in H(\operatorname{curl}, \operatorname{div} ; \Omega) ; \quad \mathbf{A} \cdot \mathbf{n}=0 \text { on } \partial \Omega\},
\end{gathered}
$$

both equipped with the norm

$$
\|\mathbf{A}\|_{H(\text { curl,div } ; \Omega)}=\left\{\|\mathbf{A}\|^{2}+\|\nabla \times \mathbf{A}\|^{2}+\|\nabla \cdot \mathbf{A}\|^{2}\right\}^{\frac{1}{2}} .
$$

As the spaces $H(\operatorname{curl}, \operatorname{div} ; \Omega)$ and $H_{0}(\operatorname{curl}, \operatorname{div} ; \Omega)$ will be frequently used, we shall write

$$
H=H(\operatorname{curl}, \operatorname{div} ; \Omega), \quad H_{0}=H_{0}(\operatorname{curl}, \operatorname{div} ; \Omega) .
$$

To treat the constraint equation $\nabla \cdot \mathbf{B}=0$, we shall need the following subspace of $H_{0}(\mathbf{c u r l}, \operatorname{div} ; \Omega)$ :

$$
V=\left\{\mathbf{A} \in H_{0}(\mathbf{c u r l}, \operatorname{div} ; \Omega) ; \quad \nabla \cdot \mathbf{A}=0 \text { in } \Omega\right\} .
$$

Due to the smoothness of the spherical domain $\Omega$, it is known that the space $H_{0}$ (curl, $\operatorname{div} ; \Omega$ ) is equivalent to the usual Sobolev space $H^{1}(\Omega)^{3}$ (see, e.g., [18]). Therefore the Sobolev space $V$ can also be written equivalently as

$$
V=\left\{\mathbf{A} \in H^{1}(\Omega)^{3} ; \quad \nabla \cdot \mathbf{A}=0 \text { in } \Omega, \mathbf{A} \cdot \mathbf{n}=0 \text { on } \partial \Omega\right\},
$$


and the following equivalence holds:

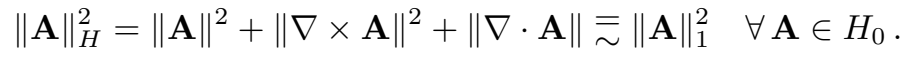

In the previous statement and what follows, $\|\cdot\|_{s, O}$ are always used to stand for the norm in Sobolev space $H^{s}(O)$ or $H^{s}(O)^{3}$ for any real number $s \geq 0$ and open bounded domain $O$. We will simply write $\|\cdot\|_{s}$ when $O=\Omega$ and $\|\cdot\|$ when $s=0$. The notation $(\cdot, \cdot)$ is used for the scalar product in $L^{2}(\Omega)$ or $L^{2}(\Omega)^{3}$, while $\langle\cdot, \cdot\rangle$ is used to denote the dual pairing between any two Hilbert spaces, and it is the extension of the scalar product $(\cdot, \cdot)$. For a nonnegative function $\beta(x)$, we will often use the notation $\|\cdot\|_{\beta}=(\beta \cdot, \cdot)^{1 / 2}$. We may also write $Q_{T}=\Omega \times(0, T)$ sometimes. In various estimates, we shall frequently use $C$ to stand for a generic constant that is independent of the mesh size $h$, time stepsize $\tau$, and relevant functions involved.

We end this subsection with a collection of some auxiliary results and formulae for later use.

(1) The space $V$ in (2.1) is a closed subspace of $H^{1}(\Omega)^{3}$; see [10].

(2) Young's inequality:

$$
a b \leq \varepsilon a^{2}+\frac{1}{4 \varepsilon} b^{2} \quad \forall a, b \in R^{1} \text { and } \varepsilon>0 .
$$

(3) Integration by parts formula which hold for $\mathbf{B} \in H(\mathbf{c u r l} ; \Omega), \mathbf{A} \in H^{1}(\Omega)^{3}$ and $q \in H^{1}(\Omega)$ :

$$
\begin{aligned}
\int_{\Omega}(\nabla \times \mathbf{B}) \cdot \mathbf{A} d \mathbf{x} & =\int_{\Omega} \mathbf{B} \cdot(\nabla \times \mathbf{A}) d \mathbf{x}-\int_{\partial \Omega}(\mathbf{B} \times \mathbf{n}) \cdot \mathbf{A} d s \\
\int_{\Omega}(\nabla \cdot \mathbf{B}) q d \mathbf{x} & =-\int_{\Omega} \mathbf{B} \cdot \nabla q d \mathbf{x}+\int_{\partial \Omega}(\mathbf{B} \cdot \mathbf{n}) q d s .
\end{aligned}
$$

(4) Compact embedding lemma [32]. Suppose that $X, B$, and $Y$ are Banach spaces satisfying $X \subset B \subset Y$ with compact embedding $X \rightarrow B$. Then for any $q>1$, each set bounded both in $L^{q}(0, T ; X)$ and $W^{1, q}(0, T ; Y)$ is relatively compact in the space $L^{q}(0, T ; B)$.

(5) Gronwall's inequality. Suppose $h(t), g(t)$ are two nonnegative and square integrable functions on $[\mathrm{a}, \mathrm{b}], c(t)$ is nondecreasing, and

$$
g(t) \leq c(t)+\int_{a}^{t} h(s) g(s) d s \quad \forall t \in[a, b],
$$

then the following holds

$$
g(t) \leq c(t) \exp \left(\int_{a}^{t} h(s) d s\right) \quad \forall t \in[a, b] .
$$

2.2. Well-posedness of the mean-field dynamo system. This section is mainly devoted to the well-posedness of the dynamo system (1.4)-(1.12). Due to the jumps in the coefficients, it is not desirable for the system to have classical type solutions. Instead, we shall seek the weak solutions to the mean-field system.

Let us first derive the variational formulation. By multiplying both sides of (1.13) by an $\mathbf{A} \in V$, integrating over $\Omega$ and making use of formula (2.3) we obtain 


$$
\begin{aligned}
& \int_{\Omega} \frac{\partial \mathbf{B}}{\partial t} \cdot \mathbf{A} d \mathbf{x}+\sum_{i=1}^{3} \int_{\Omega_{i}}(\beta \nabla \times \mathbf{B}) \cdot(\nabla \times \mathbf{A}) d \mathbf{x}-\sum_{i=1}^{3} \int_{\partial \Omega_{i}}(\beta \nabla \times \mathbf{B}) \times \mathbf{n}_{i} \cdot \mathbf{A} d s \\
= & R_{\alpha} \sum_{i=1}^{3} \int_{\Omega_{i}}\left(\frac{f}{1+\sigma|\mathbf{B}|^{2}} \mathbf{B}\right) \cdot(\nabla \times \mathbf{A}) d \mathbf{x}-R_{\alpha} \sum_{i=1}^{3} \int_{\partial \Omega_{i}}\left(\frac{f}{1+\sigma|\mathbf{B}|^{2}} \mathbf{B} \times \mathbf{n}_{i}\right) \cdot \mathbf{A} d s \\
& +R_{m} \sum_{i=1}^{3} \int_{\Omega_{i}}(\mathbf{u} \times \mathbf{B}) \cdot(\nabla \times \mathbf{A}) d \mathbf{x}-R_{m} \sum_{i=1}^{3} \int_{\partial \Omega_{i}}(\mathbf{u} \times \mathbf{B}) \times \mathbf{n}_{i} \cdot \mathbf{A} d s .
\end{aligned}
$$

Using the boundary and interface conditions (1.11) and (1.12), we deduce the variational formulation for the dynamo system (1.10)-(1.14).

Find $\mathbf{B}(t) \in V$ such that $\mathbf{B}(0)=\mathbf{B}_{0}$ and for almost all $t \in(0, T)$,

$$
\begin{aligned}
& \left(\mathbf{B}^{\prime}(t), \mathbf{A}\right)+(\beta \nabla \times \mathbf{B}(t), \nabla \times \mathbf{A}) \\
= & R_{\alpha}\left(\frac{f(t)}{1+\sigma|\mathbf{B}|^{2}} \mathbf{B}(t), \nabla \times \mathbf{A}\right)+R_{m}(\mathbf{u}(t) \times \mathbf{B}(t), \nabla \times \mathbf{A}) \quad \forall \mathbf{A} \in V,
\end{aligned}
$$

here and in what follows, functions of $\mathbf{x}$ and $t$ may be written as functions of $t$ only for simplicity.

The following theorem summarizes the well-posedness of the system (2.5).

Theorem 2.1. Assume that $\mathbf{B}_{0} \in V, f \in H^{1}\left(0, T ; L^{\infty}(\Omega)\right)$ and $\mathbf{u} \in H^{1}(0, T$; $L^{\infty}(\Omega)$ ). Then there exists a unique solution $\mathbf{B}$ to the system (2.5) with the regularity

$$
\mathbf{B} \in L^{\infty}(0, T ; V) \cap H^{1}\left(0, T ; L^{2}(\Omega)\right),
$$

and the solution $\mathbf{B}$ is stable with the following stability estimate:

$$
\begin{aligned}
\leq & C\left(\|\nabla \times \mathbf{B}(0)\|^{2}+\|\mathbf{B}(0)\|^{2}\right) \max _{0 \leq t \leq T}\left(\|f(t)\|_{L^{\infty}(\Omega)}^{2}+\|\mathbf{u}(t)\|_{L^{\infty}(\Omega)}^{2}\right) \\
& \cdot \exp \left(C \int_{0}^{T}\left\{\|f(t)\|_{L^{\infty}(\Omega)}^{2}+\left\|f^{\prime}(t)\right\|_{L^{\infty}(\Omega)}^{2}+\|\mathbf{u}(t)\|_{L^{\infty}(\Omega)}^{2}+\left\|\mathbf{u}^{\prime}(t)\right\|_{L^{\infty}(\Omega)}^{2}\right\} d t\right),
\end{aligned}
$$

where the constant $C$ depends only on the magnetic diffusivity coefficient $\beta(\mathbf{x})$.

Proof. We shall only outline the proof and refer to [10] for the details. As $H^{1}(\Omega)$ is separable, we know that $V$ is separable. Let $\left\{\mathbf{w}_{k}\right\}_{k=1}^{\infty}$ be a base of $V$, and

$$
V_{m}=\left\{\mathbf{w}_{1}, \mathbf{w}_{2}, \ldots, \mathbf{w}_{m}\right\}, \quad m=1,2,3, \ldots
$$

Choose $\psi_{m} \in V_{m}$ such that $\psi_{m} \rightarrow \mathbf{B}_{0}$ in $V$. Then we consider the following approximation of the problem (2.5): Find $\mathbf{B}_{m}(t) \in V_{m}$ such that $\mathbf{B}_{m}(0)=\psi_{m}$ and for any $\mathbf{A} \in V_{m}$,

$$
\begin{aligned}
& \left(\mathbf{B}_{m}^{\prime}(t), \mathbf{A}\right)+\left(\beta \nabla \times \mathbf{B}_{m}(t), \nabla \times \mathbf{A}\right) \\
= & R_{\alpha}\left(\frac{f}{1+\sigma\left|\mathbf{B}_{m}\right|^{2}} \mathbf{B}_{m}(t), \nabla \times \mathbf{A}\right)+R_{m}\left(\mathbf{u} \times \mathbf{B}_{m}(t), \nabla \times \mathbf{A}\right) .
\end{aligned}
$$

We claim that the sequence $\left\{\mathbf{B}_{m}(t)\right\}$ is well defined. To see this, we write

$$
\mathbf{B}_{m}(t)=\sum_{j=1}^{m} \alpha_{j, m}(t) \mathbf{w}_{j}, \quad \psi_{m}=\sum_{j=1}^{m} \gamma_{j, m} \mathbf{w}_{j}
$$


and substitute into (2.8) to get

$$
\mathcal{M} \frac{d \alpha_{m}}{d t}=\mathcal{G}\left(\alpha_{m}, t\right) \quad \text { with } \quad \alpha_{m}(0)=\gamma_{m},
$$

where $\mathcal{M}=\left(m_{i j}\right)$ with $m_{i j}=\left(\mathbf{w}_{j}, \mathbf{w}_{i}\right), \mathcal{G}\left(\alpha_{m}, t\right)$ is a vector-valued function of $\alpha_{m}$ and $t$, and

$$
\gamma_{m}(t)=\left(\gamma_{1, m}, \gamma_{2, m}, \ldots, \gamma_{m, m}\right)^{t}, \quad \alpha_{m}(t)=\left(\alpha_{1, m}, \alpha_{2, m}, \ldots, \alpha_{m, m}\right)^{t} .
$$

As $\left\{\mathbf{w}_{m}\right\}$ is linearly independent, the matrix $\mathcal{M}$ is symmetric and positive definite, so it is invertible. Then using the Lipschitz continuity of $\mathcal{G}\left(\alpha_{m}, t\right)$ with respect to $\alpha_{m}$, and our subsequent a priori estimates on the solutions to the system (2.8) that ensures the boundedness of $\mathbf{B}_{m}(t)$ independent of $m$, one can show (cf. [10]) that the solutions $\left\{\mathbf{B}_{m}(t)\right\}$ of the system $(2.8)$ is well defined in $[0, T]$.

Next, we derive some a priori estimates on the solution to (2.8). By taking $\mathbf{A}=\mathbf{B}_{m}(t)$ in (2.8), then integrating over $(0, t)$ and using the Cauchy-Schwartz and Gronwall inequality, one can obtain

$$
\begin{aligned}
& \left\|\mathbf{B}_{m}\right\|_{L^{\infty}\left(0, T ; L^{2}(\Omega)^{3}\right)}^{2}+\left\|\nabla \times \mathbf{B}_{m}\right\|_{L^{2}\left(0, T ; L^{2}(\Omega)\right)}^{2} \\
\leq & \left\|\mathbf{B}_{m}(0)\right\|_{0}^{2} \exp \left(C \int_{0}^{T}\left\{\|f(t)\|_{L^{\infty}(\Omega)}^{2}+\|\left.\mathbf{u}(t)\right|_{L^{\infty}(\Omega)} ^{2}\right\} d t\right) .
\end{aligned}
$$

On the other hand, letting $\mathbf{A}=\mathbf{B}_{m}^{\prime}(t)$ in (2.8), then integrating over $(0, t)$, applying the integration by parts and the Gronwall's inequality, we have

$$
\left\|\mathbf{B}_{m}^{\prime}\right\|_{L^{2}\left(Q_{T}\right)}^{2}+\left\|\nabla \times \mathbf{B}_{m}\right\|_{L^{\infty}\left(0, T ; L^{2}(\Omega)^{3}\right)}^{2}
$$

$$
\begin{aligned}
\leq & \|\mathbf{B}(0)\|^{2} \exp \left(C\left\{\|f\|_{L^{\infty}\left(Q_{T}\right)}^{2}+\|\left.\mathbf{u}\right|_{L^{\infty}\left(Q_{T}\right)} ^{2}\right\}\right) \\
& \cdot \exp \left(C \int_{0}^{T}\left\{\|f(t)\|_{L^{\infty}(\Omega)}^{2}+\left\|f^{\prime}(t)\right\|_{L^{\infty}(\Omega)}^{2}+\|\mathbf{u}(t)\|_{L^{\infty}(\Omega)}^{2}+\left\|\mathbf{u}^{\prime}(t)\right\|_{L^{\infty}(\Omega)}^{2}\right\} d t\right) .
\end{aligned}
$$

Using the estimates (2.10)-(2.11), we can extract a subsequence $\left\{\mathbf{B}_{n}\right\}$ from $\left\{\mathbf{B}_{m}\right\}$ such that

$$
\mathbf{B}_{n} \rightarrow \mathbf{B} \text { weakly star in } L^{\infty}(0, T ; V) ; \quad \mathbf{B}_{n}^{\prime} \rightarrow \tilde{\mathbf{B}} \text { weakly in } L^{2}\left(0, T ; L^{2}(\Omega)^{3}\right) .
$$

By the compact embedding lemma of subsection 2.1, we know that $H^{1}\left(0, T ; V^{\prime}\right) \cap$ $L^{2}(0, T ; V)$ is compactly embedded in $L^{2}\left(0, T ; L^{2}(\Omega)^{3}\right)$, so we have

$$
\mathbf{B}_{n} \rightarrow \mathbf{B} \quad \text { in } L^{2}\left(0, T ; L^{2}(\Omega)^{3}\right) .
$$

We can show that this $\mathbf{B}(t)$ solves the system (2.5). Therefore (2.5) has at least one solution $\mathbf{B}$, which has the regularity (2.6).

3. Characterization of the dynamo system in terms of a saddle-point type problem. The Sobolev space $V$ in the weak formulation (2.5) involves the solenoidal functions, and it is well known that the solenoidal conditions are difficult to enforce in finite element spaces, especially in three dimensions. Hence the variational formulation (2.5) is inconvenient and ineffective for the use in a fully discrete finite element approximation. Instead, we shall transform the variational problem (2.5) into an equivalent saddle-point type system, which can be more easily adopted for its approximations by finite element methods. 
3.1. Characterization of solenoidal functions. Let $\mathcal{D}(\Omega)$ be the set of all infinitely differentiable functions with compact supports in $\Omega$, and $\mathcal{V}$ be the subspace of $\mathcal{D}$ with all solenoidal functions:

$$
\mathcal{V}=\{\mathbf{w} \in \mathcal{D}(\Omega) ; \operatorname{div} \mathbf{w}=0 \text { in } \Omega\} .
$$

We start with the characterization of the gradient of a distribution. For any distribution function $p$ in $\Omega$, written as $p \in \mathcal{D}^{\prime}(\Omega)$, it is easy to verify that

$$
\langle\nabla p, \mathbf{w}\rangle=\sum_{i=1}^{n}\left\langle\partial_{x_{i}} p, w_{i}\right\rangle=-\sum_{i=1}^{n}\left\langle p, \partial_{x_{i}} w_{i}\right\rangle=\langle p, \nabla \cdot \mathbf{w}\rangle=0 \quad \forall \mathbf{w} \in \mathcal{V} .
$$

That is, $\nabla p$ lies in the polar set of $\mathcal{V}$. The following lemma indicates that the converse of this property is also true (cf. [18]).

Lemma 3.1. Let $\Omega$ be a bounded Lipschitz domain in $R^{n}$ and $\mathbf{f}=\left(f_{1}, f_{2}, \ldots, f_{n}\right)^{t}$ with $f_{i} \in \mathcal{D}^{\prime}(\Omega)$. Then $\mathbf{f}=\nabla p$ for some $p \in \mathcal{D}^{\prime}(\Omega)$ if and only if

$$
\langle\mathbf{f}, \mathbf{w}\rangle=0 \quad \forall \mathbf{w} \in \mathcal{V} .
$$

If $\partial_{x_{i}} p \in H^{-1}(\Omega)$, then $p \in L^{2}(\Omega)$ and

$$
\|p\|_{L^{2}(\Omega) / R} \leq C(\Omega)\|\nabla p\|_{H^{-1}(\Omega)} .
$$

Moreover, if $\partial_{x_{i}} p \in L^{2}(\Omega)$, then $p, \nabla p \in L^{2}(\Omega)$ and

$$
\|p\|_{L^{2}(\Omega) / R} \leq C(\Omega)\|\nabla p\|_{L^{2}(\Omega)} .
$$

3.2. Saddle-point formulation of the mean-field dynamo system. In this subsection, we are going to show that the solution $\mathbf{B}(t)$ of the system (2.5) also solves the following saddle-point type problem for some $p \in L^{2}\left(0, T ; L^{2}(\Omega)\right)$ :

$$
\begin{aligned}
& \frac{\partial \mathbf{B}}{\partial t}+\nabla \times(\beta(\mathbf{x}) \nabla \times \mathbf{B})+\nabla p \\
= & R_{\alpha} \nabla \times\left(\frac{f(\mathbf{x}, t)}{1+\sigma|\mathbf{B}|^{2}} \mathbf{B}\right)+R_{m} \nabla \times(\mathbf{u} \times \mathbf{B}) \quad \text { in } \Omega \times(0, T) ; \\
\nabla \cdot \mathbf{B}=0 \quad \text { in } \quad \Omega \times(0, T), &
\end{aligned}
$$

where a pressure-like term, namely a Lagrange multiplier $p$, is introduced in (3.1) to ensure that the divergence condition (3.2) is satisfied. This formulation is done purely for the convenience of the subsequent construction of some stable and convergent finite element approximations; the approach is widely employed in numerical solutions of Maxwell equations (see, e.g., [2]).

To do so, we introduce

$$
\tilde{\mathbf{B}}(t)=\int_{0}^{t} \mathbf{B}(t) d t, \quad \tilde{\mathbf{F}}(t)=\int_{0}^{t} \frac{f}{1+\sigma|\mathbf{B}|^{2}} \mathbf{B}(t) d t, \quad \tilde{\mathbf{U}}(t)=\int_{0}^{t} \mathbf{u}(t) \times \mathbf{B}(t) d t .
$$

Using the regularity of $\mathbf{B}(t)$ from Theorem 2.1 we have

$$
\tilde{\mathbf{B}}(t) \in H^{1}(0, T ; V), \quad \tilde{\mathbf{F}}(t) \in H^{1}\left(0, T ; L^{2}(\Omega)^{3}\right), \quad \tilde{\mathbf{U}}(t) \in H^{1}(0, T ; V) .
$$


Clearly, both $\tilde{\mathbf{B}}(t)$ and $\tilde{\mathbf{F}}(t)$ are absolutely continuous with respect to $t$ as $\mathbf{B}(t)$ and $f \mathbf{B}(t) /\left(1+\sigma|\mathbf{B}|^{2}\right)$ are integrable in $L^{1}(0, T)$, and we have

$$
\tilde{\mathbf{B}}^{\prime}(t)=\mathbf{B}(t), \quad \tilde{\mathbf{F}}^{\prime}(t)=\frac{f}{1+\sigma|\mathbf{B}|^{2}} \mathbf{B}(t), \quad \tilde{\mathbf{U}}^{\prime}(t)=\mathbf{u} \times \mathbf{B}(t) .
$$

Now, integrating both sides of (2.5), we obtain for all $t \in[0, T]$ and $\mathbf{A} \in V$ that

$$
\left(\mathbf{B}(t)-\mathbf{B}_{0}, \mathbf{A}\right)+(\beta \nabla \times \tilde{\mathbf{B}}(t), \nabla \times \mathbf{A})=R_{\alpha}(\tilde{\mathbf{F}}(t), \nabla \times \mathbf{A})+R_{m}(\tilde{\mathbf{U}}, \nabla \times \mathbf{A}) .
$$

We remark that this equation is defined for every $t \in[0, T]$ as $\mathbf{B}(t), \tilde{\mathbf{B}}(t)$, and $\tilde{\mathbf{F}}(t)$ are all continuous with respect to $t$. This is why we do not treat the system (2.5) directly but instead its integrated form.

For all $t \in[0, T],(3.3)$ can be written as

$$
\left\langle\mathbf{B}(t)-\mathbf{B}_{0}+\nabla \times(\beta \nabla \times \tilde{\mathbf{B}}(t))-R_{\alpha} \nabla \times \tilde{\mathbf{F}}(t)-R_{m} \nabla \times \tilde{\mathbf{U}}(t), \mathbf{A}\right\rangle=0 \quad \forall \mathbf{A} \in V,
$$

this with Lemma 3.1 indicates that there exists a $P(t) \in L^{2}(\Omega)$, for every $t \in[0, T]$, such that

$$
\mathbf{B}(t)-\mathbf{B}_{0}+\nabla \times(\beta \nabla \times \tilde{\mathbf{B}}(t))+\nabla P(t)=R_{\alpha} \nabla \times \tilde{\mathbf{F}}(t)+R_{m} \nabla \times \tilde{\mathbf{U}}(t),
$$

or we can write

$$
\nabla P(t)=\mathbf{B}_{0}-\mathbf{B}(t)-\nabla \times(\beta \nabla \times \tilde{\mathbf{B}}(t))+R_{\alpha} \nabla \times \tilde{\mathbf{F}}(t)+R_{m} \nabla \times \tilde{\mathbf{U}}(t) .
$$

Noting the right-hand side of (3.5) lies in $\left(H_{0}(\mathbf{c u r l}, \operatorname{div} ; \Omega)\right)^{\prime}$, we have

$$
\nabla P(t) \in H^{1}\left(0, T ; H_{0}(\mathbf{c u r l}, \operatorname{div} ; \Omega)^{\prime}\right) \subset H^{1}\left(0, T ; H^{-1}(\Omega)\right),
$$

then by Lemma 3.1 we obtain

$$
\|P(t)\|_{L^{2}(\Omega) / R} \leq C\|\nabla P(t)\|_{H^{-1}(\Omega)} \quad \forall t \in[0, T],
$$

this proves $P(t) \in H^{1}\left(0, T ; L^{2}(\Omega)\right)$.

Now (3.1) follows immediately by letting $p(t)=\frac{\partial P(t)}{\partial t}$ and differentiating (3.4) with respect to $t$, and $p(t) \in L^{2}\left(0, T ; L^{2}(\Omega)\right)$.

Adding a term $\gamma(\nabla \cdot \mathbf{B}, \nabla \cdot \mathbf{A})$ for some constant $\gamma>0$ in (2.5), an important stabilization term in the subsequent numerical approximation, we are then led to the following theorem.

THEOREM 3.1. The system (2.5) is equivalent to the following variational problem:

Find $\mathbf{B}(t) \in H_{0} \equiv H_{0}(\mathbf{c u r l}$, div $; \Omega)$ and $p(t) \in L_{0}^{2}(\Omega)$ such that $\mathbf{B}(0)=\mathbf{B}_{0}$ and

$$
\left\{\begin{array}{l}
\left(\mathbf{B}^{\prime}(t), \mathbf{A}\right)+(\beta \nabla \times \mathbf{B}(t), \nabla \times \mathbf{A})+\gamma(\nabla \cdot \mathbf{B}(t), \nabla \cdot \mathbf{A})+(p, \nabla \cdot \mathbf{A}) \\
=R_{\alpha}\left(\frac{f}{1+\sigma|\mathbf{B}|^{2}} \mathbf{B}(t), \nabla \times \mathbf{A}\right)+R_{m}(\mathbf{u} \times \mathbf{B}(t), \nabla \times \mathbf{A}) \quad \forall \mathbf{A} \in H_{0} \\
(\nabla \cdot \mathbf{B}, q)=0 \quad \forall q \in L_{0}^{2}(\Omega)
\end{array}\right.
$$

for a.e. $t \in(0, T)$. Moreover, we have the following stability estimates for the solution $(\mathbf{B}, p)$ :

$$
\begin{aligned}
& \|\mathbf{B}\|_{L^{\infty}(0, T ; V)}+\|\mathbf{B}\|_{H^{1}\left(0, T ; L^{2}(\Omega)^{3}\right)}+\|p\|_{L^{2}\left(0, T ; L_{0}^{2}(\Omega)\right)} \\
\leq & C\left(\|\nabla \times \mathbf{B}(0)\|^{2}+\|\mathbf{B}(0)\|^{2}\right) \max _{0 \leq t \leq T}\left(\|f(t)\|_{L^{\infty}(\Omega)}^{2}+\|\mathbf{u}(t)\|_{L^{\infty}(\Omega)}^{2}\right) \\
& \cdot \exp \left(C \int_{0}^{T}\left\{\|f(t)\|_{L^{\infty}(\Omega)}^{2}+\left\|f^{\prime}(t)\right\|_{L^{\infty}(\Omega)}^{2}+\|\mathbf{u}(t)\|_{L^{\infty}(\Omega)}^{2}+\left\|\mathbf{u}^{\prime}(t)\right\|_{L^{\infty}(\Omega)}^{2}\right\} d t\right) .
\end{aligned}
$$


Proof. From the previous derivations, we know the solution $(\mathbf{B}, p)$ to $(2.5)$ also satisfies (3.1)-(3.2). Then using the interface conditions (1.12), we can directly derive (3.7) from (3.1)-(3.2) by integration by parts. On the other hand, one can readily check by integration by parts that a solution $(\mathbf{B}, p)$ of $(3.7)$ is a solution of (3.1)-(3.2) or (2.5). This proves the equivalence of (2.5) and (3.7).

The uniqueness of the solutions to (3.7) can be done similarly to the proof of Theorem 2.1 (cf. [10]).

The estimates of the first two terms on the left-hand side of (3.8) follow from Theorem 2.1. We next derive the estimate of the last term on the left of (3.8) for $p$. For this, we introduce a $\phi \in H^{1}(\Omega) \cap L_{0}^{2}(\Omega)$ which satisfies

$$
\Delta \phi=p \quad \text { in } \Omega ; \quad \frac{\partial \phi}{\partial \mathbf{n}}=0 \quad \text { on } \partial \Omega .
$$

Then it is easy to see by Poincare's inequality that

$$
\|\nabla \phi\|^{2} \leq\|\phi\|\|p\| \leq C\|p\|\|\nabla \phi\|,
$$

which gives

$$
\|\nabla \phi\| \leq C\|p\| .
$$

Letting $b(\mathbf{A}, q)=\int_{\Omega} q \nabla \cdot \mathbf{A} d \mathbf{x}$ for any $\mathbf{A} \in H_{0}$ and $q \in L_{0}^{2}(\Omega)$, then we take a special $\mathbf{A}=\nabla \phi$. It is easy to verify that $\mathbf{A} \in H_{0}$, and

$$
\begin{aligned}
\|\mathbf{A}\|_{H_{0}(\mathbf{c u r l}, \mathrm{div} ; \Omega)} & =\left(\|\nabla \phi\|^{2}+\|p\|^{2}\right)^{\frac{1}{2}} \leq C\|p\|, \\
\frac{b(\mathbf{A}, p)}{\|\mathbf{A}\|_{H_{0}}} & =\frac{(p, p)}{\|\mathbf{A}\|_{H_{0}}} \geq C\|p\| .
\end{aligned}
$$

But we know from (3.7) that

$$
\begin{aligned}
b(\mathbf{A}, p)= & R_{\alpha}\left(\frac{f}{1+\sigma|\mathbf{B}|^{2}} \mathbf{B}(t), \nabla \times \mathbf{A}\right)+R_{m}(\mathbf{u} \times \mathbf{B}(t), \nabla \times \mathbf{A}) \\
& -\left(\mathbf{B}^{\prime}(t), \mathbf{A}\right)-(\beta \nabla \times \mathbf{B}(t), \nabla \times \mathbf{A}),
\end{aligned}
$$

from which and the Cauchy-Schwarz inequality, we obtain

$$
\begin{aligned}
b(\mathbf{A}, p) \leq & R_{\alpha}\|f(t)\|_{L^{\infty}(\Omega)}\|\mathbf{B}(t)\|\|\nabla \times \mathbf{A}\|+R_{m}\|\mathbf{u} \times \mathbf{B}(t)\|\|\nabla \times \mathbf{A}\| \\
& +\left\|\mathbf{B}^{\prime}(t)\right\|\|\mathbf{A}\|+\|\nabla \times \mathbf{B}(t)\|_{\beta}\|\nabla \times \mathbf{A}\|_{\beta} \\
\leq & C\left(\|f(t)\|_{L^{\infty}(\Omega)}\|\mathbf{B}(t)\|+\|\mathbf{u}(t)\|_{L^{\infty}(\Omega)}|\mathbf{B}(t)|+\left\|\mathbf{B}^{\prime}(t)\right\|+\|\nabla \times \mathbf{B}(t)\|\right)\|\mathbf{A}\|_{H_{0}} .
\end{aligned}
$$

Now the estimate for $p$ follows from this, (3.10), and the estimates of first two terms in (3.8).

4. Finite element approximations. In this section we shall address the finite element approximation of the nonlinear dynamo system (1.4)-(1.12), based on its saddle-point type variational formulation (3.7). As we know, the so-called edge element methods are widely used in numerical solutions of the Maxwell systems $[12,13,14]$. Their main advantages lie in the convenience to incorporate the divergence constraints implicitly and the easy satisfaction of the usual interface conditions which involve tangential components of the fields. But the nonlinear geodynamo system of our current interest requires the continuity of all the components of the magnetic field across the interfaces (see (1.12)), not just the tangential components as in other nondynamo modelling systems. This fact makes the edge element methods inconvenient for the approximation of the geodynamo system (1.4)-(1.12). Instead we shall make use of the standard Lagrange nodal finite element methods. 
4.1. Saddle-point system and its approximation. We first recall some existing well-posedness about the general saddle-point system and its approximation. Let $X$ and $M$ be two Hilbert spaces, with scalar products $(\cdot, \cdot)_{X}$ and $(\cdot, \cdot)_{M}$, respectively, and $a(v, w)$ and $b(v, q)$ be two continuous bilinear forms on $X \times X$ and $X \times M$, i.e., there exist two positive constants $\|a\|$ and $\|b\|$ such that

$$
\begin{aligned}
|a(v, w)| & \leq\|a\|\|v\|_{X}\|w\|_{X} \quad \forall v, w \in X, \\
|b(v, q)| & \leq\|b\|\|v\|_{X}\|q\|_{M} \quad \forall v \in X, q \in M .
\end{aligned}
$$

We shall need the kernel space $V$ associated with $b(\cdot, \cdot)$ and the polar set of $V$ :

$$
V=\{w \in X ; b(w, q)=0 \quad \forall q \in M\}, \quad V^{0}=\left\{g \in X^{\prime} ;\langle g, v\rangle=0 \quad \forall v \in X\right\} .
$$

Consider the saddle-point system: Find $(u, p) \in X \times M$ such that

$$
\begin{aligned}
a(u, v)+b(v, p) & =f(v) \quad \forall v \in X, \\
b(u, q) & =g(q) \quad \forall q \in M,
\end{aligned}
$$

where $f \in X^{\prime}$ and $g \in M^{\prime}$. The following well-posedness results about this saddlepoint system can be found in $[6,18]$.

Lemma 4.1. Assume (4.1) and (4.2), and

$$
\begin{aligned}
& \sup _{w \in V} \frac{a(v, w)}{\|w\|_{X}} \geq \alpha\|v\|_{X} \quad \forall v \in V ; \\
& \sup _{v \in V} a(v, w)>0 \quad \forall w \in V, w \neq 0, \\
& \sup _{v \in X} \frac{b(v, q)}{\|v\|_{X}\|q\|_{M}} \geq \beta \quad \forall q \in M, q \neq 0 .
\end{aligned}
$$

Then there exists a unique solution $(u, p) \in X \times M$ to the saddle-point problem (4.3)(4.4).

Now we discuss the approximation of the saddle-point system (4.3)-(4.4). Let $X_{h} \subset X$ and $M_{h} \subset M$ be two finite dimensional spaces, and define

$$
V_{h}=\left\{w_{h} \in X_{h} ; b\left(w_{h}, q_{h}\right)=0 \quad \forall q_{h} \in M_{h}\right\} .
$$

We then introduce a bilinear form $a_{h}(\cdot, \cdot)$ defined on $X_{h} \times M_{h}$ satisfying

$$
\begin{aligned}
a_{h}\left(v_{h}, v_{h}\right) & \geq \alpha^{*}\left\|v_{h}\right\|_{X}^{2} \quad \forall v_{h} \in V_{h}, \\
\left|a_{h}\left(v_{h}, w_{h}\right)\right| & \leq\left\|a_{h}\right\|\left\|v_{h}\right\|_{X}\left\|w_{h}\right\|_{X} \quad \forall v_{h}, w_{h} \in X_{h}
\end{aligned}
$$

for two positive constants $\alpha^{*}$ and $\left\|a_{h}\right\|$. In our later applications, $a_{h}(\cdot, \cdot)$ comes from some approximation of $a(\cdot, \cdot)$ and is formed from $a(\cdot, \cdot)$ in such a way that numerical integrations on polyhedra with curved faces are replaced by much easier integrations on polyhedra with planar faces.

Then we introduce the approximation of the saddle-point system (4.3)-(4.4).

Find $\left(u_{h}, p_{h}\right) \in X_{h} \times M_{h}$ such that

$$
\begin{aligned}
a_{h}\left(u_{h}, v_{h}\right)+b\left(v_{h}, p_{h}\right) & =f\left(v_{h}\right) \quad \forall v_{h} \in X_{h} \\
b\left(u_{h}, q_{h}\right) & =g\left(q_{h}\right) \quad \forall q_{h} \in M_{h} .
\end{aligned}
$$


We have the following convergence theorem (cf. [6]), whose detailed proof can be found in [10].

THEOREM 4.1. In addition to the assumptions (4.8)-(4.9), we assume that the inf-sup condition

$$
\sup _{v_{h} \in X_{h}} \frac{b\left(v_{h}, q_{h}\right)}{\left\|v_{h}\right\|_{X}\left\|q_{h}\right\|_{M}} \geq \beta^{*} \quad \forall q_{h} \in M_{h}, q_{h} \neq 0 .
$$

is also satisfied. Then the system (4.10)-(4.11) has a unique solution $\left(u_{h}, p_{h}\right) \in$ $X_{h} \times M_{h}$ and the following error estimate holds:

$$
\begin{aligned}
\left\|u-u_{h}\right\|_{X} \leq & \left(1+\frac{\left\|a_{h}\right\|}{\alpha^{*}}\right)\left(1+\frac{\|b\|}{\beta^{*}}\right) \inf _{v_{h} \in X_{h}}\left\|u-v_{h}\right\|_{X}+\|b\| \inf _{\mu_{h} \in M_{h}}\left\|p-\mu_{h}\right\|_{M} \\
& +\frac{1}{\alpha^{*}} \sup _{v_{h} \in V_{h}} \frac{a\left(u, v_{h}\right)-a_{h}\left(u, v_{h}\right)}{\left\|v_{h}\right\|_{X}} .
\end{aligned}
$$

4.2. A fully discrete finite element method and its stability. In this section, we will propose a fully discrete finite element method for the variational system (3.7). For this purpose, we have to approximate the problem in both time and space. We shall use the backward Euler scheme for time discretization and the popular Hood-Taylor finite elements (cf. [20]) for space discretization.

We start with the partition of the time interval $[0, \mathrm{~T}]$ and the triangulation of the physical spherical domain $\Omega$. We divide the time interval $[0, \mathrm{~T}]$ into $M$ equally spaced subintervals using the following nodal points:

$$
0=t_{0}<t_{1}<t_{2}<\cdots<t_{M}=\mathrm{T}
$$

where $t_{n}=n \tau$ for $n=0,1, \ldots, M$ and $\tau=T / M$. For any given discrete time sequence $\left\{u^{n}\right\}_{n=0}^{M}$ with each $u^{n}$ lying in $L^{2}(\Omega)$ or $L^{2}(\Omega)^{3}$, we define the first order backward finite differences and the averages as follows:

$$
\partial_{\tau} u^{n}=\frac{u^{n}-u^{n-1}}{\tau}, \quad \bar{u}^{n}=\frac{1}{\tau} \int_{t_{n-1}}^{t_{n}} u(\cdot, s) d s .
$$

If $u(\mathbf{x}, t)$ is a function which is continuous with respect to $t$, we shall often write $u^{n}(\cdot)=u\left(\cdot, t_{n}\right)$ for $n=0,1, \ldots, M$. For the ease of exposition, we may also use the function values for $t \leq 0$, by assuming the convention that $u(\mathbf{x}, t)=u(\mathbf{x}, 0)$ for all $t \leq 0$.

We now introduce the triangulation of the domain $\Omega$, consisting of the inner core $\Omega_{1}$, the outer core $\Omega_{2}$, and the exterior zone $\Omega_{3}$. For the sake of technical treatments, we shall assume that the outer boundary of the exterior zone $\Omega_{3}$ is a closed convex polygon; the actual curved boundary case can be treated in the same manner as we handle in this and next section the curved interfaces $\Gamma_{1}$ and $\Gamma_{2}$; see Figure 1.

We first triangulate the inner core $\Omega_{1}$ using a quasi-uniform triangulation $\mathcal{T}_{h}^{1}$ with tetrahedral elements of mesh size $h$, which form a polyhedral domain $\Omega_{h}^{1} \subset \Omega_{1}$. The triangulation is done such that the boundary vertices of $\Omega_{h}^{1}$ all lie on the boundary of $\Omega_{1}$.

Then we triangulate the exterior zone $\Omega_{3}$ using a triangulation $\mathcal{T}_{h}^{3}$ with tetrahedral elements, which form a polyhedral domain $\Omega_{h}^{3}$. The triangulation is done such that all the vertices on the outer polygonal boundary $\partial \Omega$ are also vertices of $\Omega_{h}^{3}$, and the inner boundary vertices of $\Omega_{h}^{3}$ all lie on the inner boundary of $\Omega_{3}$. 
Finally we triangulate the outer core $\Omega_{2}$ using a triangulation $\mathcal{T}_{h}^{2}$ with tetrahedral elements, which form a polyhedral domain $\Omega_{h}^{2}$. The triangulation is done such that all the vertices on the outer boundary of $\Omega_{h}^{2}$ match those vertices on the inner boundary of $\Omega_{h}^{3}$, while all the vertices on the inner boundary of $\Omega_{h}^{2}$ match the boundary vertices of $\Omega_{h}^{1}$.

Now the three individual triangulations $\mathcal{T}_{h}^{1}, \mathcal{T}_{h}^{2}$, and $\mathcal{T}_{h}^{3}$ form a global triangulation $\mathcal{T}_{h}$ of $\Omega$. By $\mathcal{N}_{h}$ we shall denote the set of all the nodal points of the triangulation $\mathcal{T}_{h}$, and by $\mathcal{F}_{h}$ the set of all faces of elements in $\mathcal{T}_{h}$.

For convenience, any element $K$ of $\mathcal{T}_{h}$ whose interior has nonempty intersection with the interface $\Gamma_{1}$ and $\Gamma_{2}$ will be called an interface element. The set of all interface elements is denoted by $\mathcal{T}_{h}^{*}$. Let us introduce some notation needed in the subsequent error estimates. For each interface element $K \in \mathcal{T}_{h}^{*}$, we know that $K$ must lie either in $\Omega_{2}^{h}$ or $\Omega_{3}^{h}$ according to the construction of the triangulation $\mathcal{T}_{h}$. And each interface element $K$ is divided by the interface into two parts, written as $\mathcal{K}_{1}$ and $\mathcal{K}_{2}$. Since the interfaces $\Gamma_{1}$ and $\Gamma_{2}$ are smooth spheric surfaces, one can show (cf. [17]) that one of the two parts $\mathcal{K}_{1}$ and $\mathcal{K}_{2}$, denoted always by $\mathcal{K}$, has a volume of order $h_{K}^{4}$, that is,

$$
|\mathcal{K}| \equiv h_{K}^{4} .
$$

Here and in what follows, we shall often use the symbols $\lesssim$ and $\overline{\bar{\sim}}$, and $x \lesssim y$ means that $x \leq C y$ for some generic constant $C$, and $x \approx y$ means $x \lesssim y$ and $y \lesssim x$.

Also we may absorb in the generic constant $C$ the upper and lower bounds $\beta_{m}$, $\beta_{M}, f_{M}$, and $u_{M}$ of the functions $\beta(\mathbf{x}), f(\mathbf{x}, t)$, and $\mathbf{u}(\mathbf{x}, t)$ over $\Omega \times(0, T)$ :

$$
\beta_{m} \leq \beta(\mathbf{x}) \leq \beta_{M} ; \quad|f(\mathbf{x}, t)|,\left|f_{t}(\mathbf{x}, t)\right| \leq f_{M} ; \quad|\mathbf{u}(\mathbf{x}, t)|,\left|\mathbf{u}_{t}(\mathbf{x}, t)\right| \leq u_{M} .
$$

Noting the coefficient $\beta(\mathbf{x})$ in (1.13) has large jumps across the interfaces $\Gamma_{1}$ and $\Gamma_{2}$, hence it may be strongly discontinuous inside each interface element $K \in \mathcal{T}_{h}^{*}$, namely when crossing the (curved) common face of two curved polyhedra parts $\mathcal{K}_{1}$ and $\mathcal{K}_{2}$ of $K$. To avoid numerical integrations on polyhedra with curved faces in forming the finite element stiffness matrix, we introduce the following approximations of the coefficients $\beta(\mathbf{x}), f(\mathbf{x}, t)$, and $\mathbf{u}(\mathbf{x}, t)$ :

$$
\begin{gathered}
\beta_{h}(\mathbf{x})=\beta(\mathbf{x}), \mathbf{x} \in K \in \mathcal{T}_{h} \backslash \mathcal{T}_{h}^{*} ; \quad \beta_{h}(\mathbf{x})=\beta_{i}(\mathbf{x}), \mathbf{x} \in K \in \mathcal{T}_{h}^{*} \cap \Omega_{i}^{h}(i=2 \text { or } 3), \\
f_{h}(\mathbf{x}, t)=\left\{\begin{array}{cc}
0, & \mathbf{x} \in K \in \mathcal{T}_{h}^{*} \cap \Omega_{3}^{h} ; \\
f(\mathbf{x}, t), & \text { otherwise }
\end{array} \quad \mathbf{u}_{h}(\mathbf{x}, t)=\left\{\begin{array}{cc}
0, & \mathbf{x} \in K \in \mathcal{T}_{h}^{*} \cap \Omega_{3}^{h} \\
\mathbf{u}(\mathbf{x}, t), & \text { otherwise }
\end{array}\right.\right.
\end{gathered}
$$

We shall use the Hood-Taylor finite elements (cf. [18, 15, 33]) to approximate the system (3.7), namely the piecewise quadratic polynomials for the magnetic field $\mathbf{B}$ and the piecewise linear polynomials for the Lagrange multiplier $p$. These spaces can be defined as follows:

$$
\begin{aligned}
H_{h} & =\left\{\mathbf{w} \in C(\bar{\Omega}):\left.\mathbf{w}\right|_{K} \in P_{2}(K)^{3} \forall K \in \mathcal{T}_{h}\right\}, \\
H_{0 h} & =\left\{\mathbf{w} \in H_{h} ; \mathbf{w} \cdot \mathbf{n}_{F}=0 \forall F \in \mathcal{F}_{h} \cap \partial \Omega\right\}, \\
Q_{h} & =\left\{q_{h} \in C(\bar{\Omega}) ;\left.q_{h}\right|_{K} \in P_{1}(K) \forall K \in \mathcal{T}_{h}\right\},
\end{aligned}
$$

where $\mathbf{n}_{F}$ is the unit normal vector of a face $F \in \mathcal{F}_{h}$. And the following subspaces of $H_{0 h}$ and $Q_{h}$ will be also needed:

$$
\tilde{H}_{0 h}=\left\{\mathbf{w}_{h} \in H_{0 h} ; \mathbf{w}_{h}=0 \text { on } \partial \Omega\right\}, \quad Q_{0 h}=\left\{q_{h} \in Q_{h} ; \int_{\Omega} q_{h} d x=0\right\} .
$$


Now, we are ready to propose the fully discrete finite element approximation of the variational problem (3.7) using the approximate functions $\beta_{h}, f_{h}$, and $\mathbf{u}_{h}$.

Find $\mathbf{B}_{h}^{n} \in H_{0 h}, p_{h}^{n} \in Q_{0 h}$ for $n=1,2, \ldots, M$ such that $\mathbf{B}_{h}^{0}=S_{h} \mathbf{B}_{0}$ and

$$
\left\{\begin{array}{l}
\left(\partial_{\tau} \mathbf{B}_{h}^{n}, \mathbf{A}_{h}\right)+\left(\beta_{h} \nabla \times \mathbf{B}_{h}^{n}, \nabla \times \mathbf{A}_{h}\right)+\gamma\left(\nabla \cdot \mathbf{B}_{h}^{n}, \nabla \cdot \mathbf{A}_{h}\right)+\left(p_{h}^{n}, \nabla \cdot \mathbf{A}_{h}\right) \\
\quad=R_{\alpha}\left(\frac{f_{h}^{n}}{1+\sigma\left|\mathbf{B}_{h}^{n-1}\right|^{2}} \mathbf{B}_{h}^{n}, \nabla \times \mathbf{A}_{h}\right)+R_{m}\left(\mathbf{u}_{h}^{n} \times \mathbf{B}_{h}^{n}, \nabla \times \mathbf{A}_{h}\right) \quad \forall \mathbf{A}_{h} \in H_{0 h} \\
\left(\nabla \cdot \mathbf{B}_{h}^{n}, q_{h}\right)=0 \quad \forall q_{h} \in Q_{0 h},
\end{array}\right.
$$

where $S_{h}$ is the modified Scott-Zhang interpolation to be defined in section 5 . One may replace $S_{h}$ here by the computationally less expensive standard interpolation operator $\Pi_{h}$ induced by the finite element space $H_{h}$, but as it will be seen in the subsequent analysis, with $\Pi_{h}$ one requires a stronger regularity on the initial data $\mathbf{B}_{0}$.

We remark that the discrete system (4.15) cannot ensure $\nabla \cdot \mathbf{B}_{h}^{n}=0$, different from the continuous case. The next lemma verifies the well-posedness of the fully discrete scheme (4.15).

LEMMA 4.2. There exists a unique solution $\left(\mathbf{B}_{h}^{n}, p_{h}^{n}\right)$ to the discrete system (4.15) for each fixed $n(1 \leq n \leq M)$ and the sequence $\left\{\mathbf{B}_{h}^{n}\right\}_{n=0}^{M}$ has the following stability estimates:

$$
\max _{1 \leq n \leq M}\left\|\mathbf{B}_{h}^{n}\right\|^{2}+\tau \sum_{n=1}^{M}\left(\left\|\nabla \times \mathbf{B}_{h}^{n}\right\|^{2}+\left\|\nabla \cdot \mathbf{B}_{h}^{n}\right\|^{2}\right) \lesssim\left\|\mathbf{B}_{h}^{0}\right\|^{2} .
$$

Proof. Inequality (4.16) follows by taking $\mathbf{A}_{h}=\tau \mathbf{B}_{h}^{n}$ in (4.15) and the discrete Gronwall's inequality.

We now verify the existence of solutions to (4.15) for each fixed $n=T / M$ and $h$ by applying the Brouwer fixed point theorem. To this aim, we define a mapping $F_{h}:\left(\overline{\mathbf{B}}_{h}, \bar{p}_{h}\right) \rightarrow\left(\mathbf{B}_{h}, p_{h}\right)$ by

$$
\left\{\begin{array}{l}
\tilde{a}_{h}\left(\mathbf{B}_{h}, \mathbf{A}_{h}\right)+\tilde{b}\left(\mathbf{A}_{h}, p_{h}\right)=\tilde{g}\left(\overline{\mathbf{B}}_{h}, \mathbf{A}_{h}\right) \quad \forall \mathbf{A}_{h} \in H_{0 h} \\
\tilde{b}\left(\mathbf{B}_{h}, q_{h}\right)=0 \quad \forall q_{h} \in Q_{0 h}
\end{array}\right.
$$

where $\tilde{a}_{h}, \tilde{b}$ and $\tilde{g}$ are given by

$$
\begin{aligned}
\tilde{a}_{h}(\mathbf{B}, \mathbf{A}) & =(\mathbf{B}, \mathbf{A})+\tau\left(\beta_{h} \nabla \times \mathbf{B}, \nabla \times \mathbf{A}\right)+\gamma \tau(\nabla \cdot \mathbf{B}, \nabla \cdot \mathbf{A}), \tilde{b}(\mathbf{A}, q)=\tau(q, \nabla \cdot \mathbf{A}), \\
\tilde{g}(\mathbf{B}, \mathbf{A}) & =\left(\mathbf{B}_{h}^{n-1}, \mathbf{A}\right)+\tau R_{\alpha}\left(\frac{f_{h}^{n}}{1+\sigma\left|\mathbf{B}_{h}^{n-1}\right|^{2}} \mathbf{B}, \nabla \times \mathbf{A}\right)+\tau R_{m}\left(\mathbf{u}_{h}^{n} \times \mathbf{B}, \nabla \times \mathbf{A}\right) .
\end{aligned}
$$

By applying Theorem 4.1 one can show that the mapping $F_{h}$ is well defined; see [10] for details.

We next show that $F_{h}$ maps a bounded subset of $H_{0 h} \times Q_{0 h}$ into itself. In fact, taking $\mathbf{A}_{h}=\mathbf{B}_{h}$ in the first equation of (4.17), using the second equation and Young's inequality we can obtain

$$
\left\|\mathbf{B}_{h}\right\|^{2}+\tau\left\|\nabla \times \mathbf{B}_{h}\right\|_{\beta_{h}}^{2}+\gamma \tau\left\|\nabla \cdot \mathbf{B}_{h}\right\|^{2} \leq\left\|\mathbf{B}_{h}^{n-1}\right\|^{2}+\frac{2 \tau}{\beta_{m}}\left(R_{\alpha}^{2} f_{M}^{2}+4 R_{m}^{2} u_{M}^{2}\right)\left\|\overline{\mathbf{B}}_{h}\right\|^{2} .
$$

Thus for any $\overline{\mathbf{B}}_{h}$ lying in the ball $B\left(0, r_{0}\right)=\left\{\mathbf{A}_{h} ;\left\|\mathbf{A}_{h}\right\|_{H_{0}} \leq r_{0}\right\}$ with $r_{0}=$ $\sqrt{2}\left\|\mathbf{B}_{h}^{n-1}\right\|$, we have

$$
\left\|\mathbf{B}_{h}\right\|^{2}+\tau\left\|\nabla \times \mathbf{B}_{h}\right\|_{\beta_{h}}^{2}+\gamma \tau\left\|\nabla \cdot \mathbf{B}_{h}\right\|^{2} \leq r_{0}^{2}
$$


when $\tau$ is appropriately small such that $4 \tau\left(R_{\alpha}^{2} f_{M}^{2}+4 R_{m}^{2} u_{M}^{2}\right) \leq \beta_{m}$. Next we show that $p_{h}$ lies in the ball $B\left(0, \bar{r}_{0}\right)$ with

$$
\bar{r}_{0}=C_{0}^{-1}\left(\frac{2}{\tau}+\frac{\sqrt{\gamma}+\sqrt{\beta_{M}}}{\sqrt{\tau}}+R_{\alpha} f_{M}+2 R_{m} u_{M}\right) r_{0} .
$$

To see this, for any $\mathbf{A}_{h} \in H_{0 h}$, we obtain from (4.17) using the Cauchy-Schwarz inequality that

$$
\begin{aligned}
\tau\left(\nabla \cdot \mathbf{A}_{h}, p_{h}\right) \leq & \left(\left\|\mathbf{B}_{h}\right\|+\tau \beta_{M}^{1 / 2}\left\|\nabla \times \mathbf{B}_{h}\right\|_{\beta}+\gamma \tau\left\|\nabla \cdot \mathbf{B}_{h}\right\|\right. \\
& \left.+\left\|\mathbf{B}_{h}^{n-1}\right\|+\tau R_{\alpha} f_{M}\left\|\overline{\mathbf{B}}_{h}\right\|+2 \tau R_{m} u_{M}\left\|\overline{\mathbf{B}}_{h}\right\|\right)\left\|\mathbf{A}_{h}\right\|_{H_{0}} .
\end{aligned}
$$

This, combined with the inf-sup condition for $\tilde{b}(\cdot, \cdot)$ (cf. [10]), leads to the conclusion that $p_{h}$ lies in the ball $B\left(0, \bar{r}_{0}\right)$. Thus we have proved that $F_{h}$ maps the bounded subset $B\left(0, r_{0}\right) \times B\left(0, \bar{r}_{0}\right)$ of $H_{0 h} \times Q_{0 h}$ into itself. Therefore by the Brouwer fixed point theorem, $F_{h}$ has a fixed point $\left(\mathbf{B}_{h}, p_{h}\right) \in B\left(0, \bar{r}_{0}\right) \times B\left(0, \bar{r}_{0}\right)$. This proves the existence of solutions to the system (4.15).

The uniqueness of the solutions can be shown in the same manner as in Theorem 3.1.

5. Convergence analysis of the fully discrete finite element method. This section will be devoted to the convergence analysis on the fully discrete finite element approximation (4.15) to the variational problem (3.7). As we shall see, one of the crucial tools in the analysis relies on the following projection operator $P_{h}$ which maps functions from the space $H_{0} \times Q_{0} \equiv H_{0}(\mathbf{c u r l}$, div; $\Omega) \times L_{0}^{2}(\Omega)$ into $H_{0 h} \times Q_{0 h}$ : for any $(\mathbf{B}, p) \in H_{0} \times Q_{0},\left(\mathbf{B}_{h}, p_{h}\right)=P_{h}(\mathbf{B}, p) \in H_{0 h} \times Q_{0 h}$ solves the following saddle-point system:

$$
\left\{\begin{array}{c}
\left(\mathbf{B}_{h}, \mathbf{A}_{h}\right)+a_{h}\left(\mathbf{B}_{h}, \mathbf{A}_{h}\right)+\left(p_{h}, \nabla \cdot \mathbf{A}_{h}\right) \\
=\left(\mathbf{B}, \mathbf{A}_{h}\right)+a\left(\mathbf{B}, \mathbf{A}_{h}\right)+\left(p, \nabla \cdot \mathbf{A}_{h}\right) \quad \forall \mathbf{A}_{h} \in H_{0 h}, \\
\left(\nabla \cdot \mathbf{B}_{h}, q_{h}\right)=0 \quad \forall q_{h} \in Q_{0 h},
\end{array}\right.
$$

where for any $\mathbf{B}, \mathbf{A} \in H_{0}, a(\mathbf{B}, \mathbf{A})$ and $a_{h}(\mathbf{B}, \mathbf{A})$ are given by

$$
\begin{aligned}
a(\mathbf{B}, \mathbf{A}) & =(\beta \nabla \times \mathbf{B}, \nabla \times \mathbf{A})+\gamma(\nabla \cdot \mathbf{B}, \nabla \cdot \mathbf{A}), \\
a_{h}(\mathbf{B}, \mathbf{A}) & =\left(\beta_{h} \nabla \times \mathbf{B}, \nabla \times \mathbf{A}\right)+\gamma(\nabla \cdot \mathbf{B}, \nabla \cdot \mathbf{A}) .
\end{aligned}
$$

By taking $\mathbf{A}_{h}=\mathbf{B}_{h}$ in (5.1) and using Young's inequality and the bounds of $\beta(\mathbf{x})$ and $\beta_{h}(\mathbf{x})$, we can directly establish the following stability estimates on the projection $P_{h}$ (cf. [10]):

Lemma 5.1. For any $\mathbf{B} \in H_{0}$ and $p \in Q_{0}$, let $\left(\mathbf{B}_{h}, p_{h}\right)$ be the projection of $(\mathbf{B}, p)$ defined by (5.1), then we have

$$
\left\|\mathbf{B}_{h}\right\|_{H_{0}(\mathbf{c u r l}, \mathrm{div} ; \Omega)} \lesssim\|\mathbf{B}\|_{H_{0}(\mathbf{c u r l}, \mathrm{div} ; \Omega)}+\|p\| .
$$

Considering the discontinuity of coefficient $\beta(\mathbf{x})$ across the interfaces $\Gamma_{1}$ and $\Gamma_{2}$, the solution $(\mathbf{B}, p)$ to the system (3.7) often has higher regularity locally inside each medium subdomain $\Omega_{i}(i=1,2,3)$ than in the entire domain $\Omega$. To make full use of the better local regularities of $(\mathbf{B}, p)$ to establish the error estimates of the projection $P_{h}$, we can introduce a specially constructed interpolation operator by modifying the 
Scott-Zhang operator [29] such that it preserves the boundary condition in $H_{0}$ : for any $\mathbf{B} \in H_{0}$, we have $S_{h} \mathbf{B} \in H_{0 h}$ (see [10] for details); and $S_{h}$ has the following local approximation property (cf. $[29,10])$ :

$$
\left\|w-S_{h} w\right\|_{W^{m, p}(K)} \lesssim h_{K}^{l-m}\|w\|_{W^{l, p}\left(S_{K}\right)} \quad \forall w \in W^{l, p}\left(S_{K}\right),
$$

where $0<m \leq l$ and $S_{K}$ is the union of all elements in $\mathcal{T}_{h}$, whose closure has nonempty intersection with $K$. We now establish the error estimates of form (5.2) in the entire domain $\Omega$ for functions with higher regularities locally in each subdomain $\Omega_{k}(k=1,2,3)$.

Lemma 5.2. For any $s \geq 0$, and $u \in X=H^{1}(\Omega) \cap H^{1+s}\left(\Omega_{k}\right)(k=1,2,3)$,

$$
\left\|u-S_{h} u\right\| \lesssim h^{1+\frac{2 s}{3}} \sum_{k=1}^{3}\|u\|_{1+s, \Omega_{k}}, \quad\left\|u-S_{h} u\right\|_{1} \lesssim h^{\frac{2 s}{3}} \sum_{k=1}^{3}\|u\|_{1+s, \Omega_{k}} .
$$

Proof. For any $u \in X$, let $u_{k}$ be the restriction of $u$ on $\Omega_{k}(k=1,2,3)$. Noting the interfaces $\Gamma_{1}$ and $\Gamma_{2}$ are smooth, one can extend (cf. [30]) $u_{k} \in H^{1+s}\left(\Omega_{k}\right)$ onto the whole domain $\Omega$ such that the extended function $\tilde{u}_{k} \in H^{1+s}(\Omega)$ and

$$
\left\|\tilde{u}_{k}\right\|_{1+s, \Omega} \lesssim\left\|u_{k}\right\|_{1+s, \Omega_{k}} \quad \text { for } k=1,2,3 .
$$

First, we consider the estimate on any noninterface element $K \notin \mathcal{T}_{h}^{*}$. Since $u$ has $H^{1+s}$-regularity in such element $K$, one can follow the standard error estimate in [29] and make use of our construction of the face $\tau_{i}$ associated with each node $a_{i}$ to derive

$$
\left\|u-S_{h} u\right\|_{\mu, K} \lesssim h^{1+s-\mu} \sum_{i=1}^{3}\|u\|_{1+s, S_{K} \cap \Omega_{i}}, \quad \mu=0,1 .
$$

The tricky case happens to the interface elements. Without loss of generality, consider an interface element $K \in \mathcal{T}_{h}^{*}$ near the interface $\Gamma_{1}$. We analyze the errors in $\mathcal{K}_{1}$ and $\mathcal{K}_{2}$ separately. Clearly, $\mathcal{K}_{1} \subset \Omega_{1}, \mathcal{K}_{2} \subset \Omega_{2},\left|\mathcal{K}_{1}\right| \equiv h_{K}^{4}$ by (4.14). Then by Hölder's inequality, Sobolev embedding, and (5.2), we derive for any $2 \leq p \leq 6 /(3-2 s)$ and $\mu=0,1$ that

$$
\begin{aligned}
\left\|u-S_{h} u\right\|_{\mu, \mathcal{K}_{1}}^{2} & \lesssim h_{K}^{\frac{4(p-2)}{p}}\left\|u-S_{h} u\right\|_{W^{\mu, p}\left(\mathcal{K}_{1}\right)}^{2} \\
& \lesssim h_{K}^{\frac{4(p-2)}{p}}\left\|u-S_{h} u\right\|_{W^{\mu, p}(K)}^{2} \lesssim h_{K}^{6-2 \mu-\frac{8}{p}}\|u\|_{W^{1, p}\left(S_{K}\right)}^{2} .
\end{aligned}
$$

But on $\mathcal{K}_{2}$, by the choice of the face $\tau_{i}$ associated with the node $a_{i}$ in the definition of $S_{h}$ we know that

$$
\tilde{u}_{2}=u_{2} \text { on } \mathcal{K}_{2}, \quad S_{h} \tilde{u}_{2}=S_{h} u \text { on } \mathcal{K}_{2} .
$$

Using this and (5.2), we derive

$$
\left\|u-S_{h} u\right\|_{\mu, \mathcal{K}_{2}}^{2} \lesssim\left\|\tilde{u}_{2}-S_{h} \tilde{u}_{2}\right\|_{\mu, \mathcal{K}_{2}}^{2} \lesssim\left\|\tilde{u}_{2}-S_{h} \tilde{u}_{2}\right\|_{\mu, K}^{2} \lesssim h_{K}^{2(1+s-\mu)}\left\|\tilde{u}_{2}\right\|_{1+s, S_{K}}^{2},
$$

combined with the previous estimate on $\mathcal{K}_{1}$ and (5.3) yields

$$
\sum_{K \in \mathcal{T}_{h}^{*}}\left\|u-S_{h} u\right\|_{\mu, K}^{2} \lesssim h^{2(1+s-\mu)} \sum_{k=1}^{3}\left\|\tilde{u}_{k}\right\|_{1+s, \Omega_{k}}^{2}+\sum_{K \in \mathcal{T}_{h}^{*}} h_{K}^{6-2 \mu-\frac{8}{p}}\|u\|_{W^{1, p}\left(S_{K}\right)}^{2} .
$$


Then by Hölder's inequality and the fact that the number of interface elements in $\mathcal{T}_{h}^{*} \lesssim h^{-2}$

$$
\sum_{K \in \mathcal{T}_{h}^{*}}\left\|u-S_{h} u\right\|_{\mu, K}^{2} \lesssim h^{2(1+s-\mu)} \sum_{k=1}^{3}\|u\|_{1+s, \Omega_{k}}^{2}+h^{4-2 \mu-\frac{4}{p}}\left(\sum_{K \in \mathcal{T}_{h}^{*}}\|u\|_{W^{1, p}\left(S_{K}\right)}^{p}\right)^{2 / p} .
$$

Now the desired estimate follows by taking $p=6 /(3-2 s)$ above and using (5.4).

The following lemma provides a crucial observation needed in the subsequent analysis.

LEMMA 5.3. Let $\mathcal{K}$ be the interface part of any interface element $K \in \mathcal{T}_{h}^{*}$ such that $|\mathcal{K}| \equiv h_{K}^{4}$ (cf. (4.14)), then the following estimates hold:

$$
\left\|\nabla \times \mathbf{A}_{h}\right\|_{0, \mathcal{K}}^{2} \lesssim h_{K}\left\|\nabla \times \mathbf{A}_{h}\right\|_{0, K}^{2}, \quad\left\|\mathbf{A}_{h}\right\|_{0, \mathcal{K}}^{2} \lesssim h_{K}\left\|\mathbf{A}_{h}\right\|_{0, K}^{2} \quad \forall \mathbf{A}_{h} \in H_{0 h} .
$$

Proof. We prove only the first inequality in (5.7), the second is similar. Let $K$ be an interface element with 4 vertices, $v_{1}, v_{2}, v_{3}$, and $v_{4}$, and $d_{K}$ be the largest distance from the curved side of $\mathcal{K}$ to its opposite face of $K$. Since the interfaces $\Gamma_{1}$ and $\Gamma_{2}$ are $C^{\infty}$-smooth, we can easily show that $d_{K} \leq C h_{K}^{2}$. Then we can construct a cube $\mathcal{C}(\mathcal{K})$ such that $\mathcal{K} \subset C(\mathcal{K})$ and $\mathcal{C}(\mathcal{K})$ has a height $d_{K}$ and a rectangular base of length $\alpha_{1} h_{K}$ and width $\alpha_{2} h_{K}$, where $\alpha_{1}$ and $\alpha_{2}$ are two positive constants independent of mesh size $h$. Then we divide $\mathcal{C}(\mathcal{K})$ into 6 small tetrahedra $\mathcal{K}^{1}, \ldots, \mathcal{K}^{6}$.

By scaling arguments, one can easily verify the equivalence

$$
\|q\|_{0, A}^{2}=|A| \sum_{i=1}^{4}\left(q\left(a_{i}\right)\right)^{2} \quad \forall q \in P_{1}(A)
$$

for any tetrahedron $A$ with vertices $a_{1}, a_{2}, a_{3}$, and $a_{4}$. Let $p$ be a component of $\nabla \times \mathbf{A}_{h}$ for some $\mathbf{A}_{h} \in H_{0 h}$, then $p \in P_{1}(K)$. Clearly we also see $p \in P_{1}(\mathcal{K})$, and $p$ can be naturally extended to $\tilde{p} \in P_{1}(\mathcal{C}(\mathcal{K}))$, thus

$$
\|p\|_{0, \mathcal{K}}^{2} \leq\|\tilde{p}\|_{0, \mathcal{C}(\mathcal{K})}^{2} \leq \sum_{i=1}^{6}\|\tilde{p}\|_{0, \mathcal{K}^{i}}^{2}
$$

But using (5.8) and the fact that the value $\tilde{p}$ at any point in $\mathcal{K}^{i}$ can be expressed as a convex combination of the values of $p$ at the 4 vertices of $K$, we obtain

$$
\|p\|_{0, \mathcal{K}}^{2} \lesssim h_{K}^{4} \sum_{j=1}^{4}\left(p\left(v_{j}\right)\right)^{2} \approx h_{K}|K| \sum_{j=1}^{4}\left(p\left(v_{j}\right)\right)^{2} \approx h_{K}\|p\|_{0, K}^{2}
$$

This proves the first estimate in (5.7).

Using the interpolation error estimates in Lemma 5.2 and the convergence theory in Theorem 4.1, we can now derive the error estimate for the projection operator $P_{h}$ defined in (5.1).

Lemma 5.4. Let $\mathbf{B} \in H_{0}(\mathbf{c u r l}, \operatorname{div} ; \Omega)$ and $p \in L_{0}^{2}(\Omega)$ be given such that $\mathbf{B} \in$ $H^{1+s_{1}}\left(\Omega_{k}\right)$ in each $\Omega_{k}(k=1,2,3)$ for some $0 \leq s_{1}<1$ and $p \in H^{s_{2}}(\Omega)$ for some $0 \leq s_{2}<1$. Then the following error estimates hold for the projection $\left(\mathbf{B}_{h}, p_{h}\right)$ of $(\mathbf{B}, p)$ defined in (5.1):

$$
\sum_{i=1}^{3}\left\|\mathbf{B}-\mathbf{B}_{h}\right\|_{H\left(\text { curl }, \mathrm{div} ; \Omega_{i}\right)}^{2} \lesssim h^{\frac{4 s_{1}}{3}} \sum_{i=1}^{3}\|\mathbf{B}\|_{1+s_{1}, \Omega_{i}}^{2}+h^{2 s_{2}}\|p\|_{s_{2}, \Omega}^{2} .
$$


Proof. Let $X=H_{0}(\mathbf{c u r l}, \operatorname{div} ; \Omega)$ and $M=L_{0}^{2}(\Omega), X_{h}=H_{0 h}$, and $M_{h}=Q_{0 h}$, then we can apply Theorem 4.1 to the system (5.1) to obtain

$$
\begin{gathered}
\left\|\mathbf{B}-\mathbf{B}_{h}\right\|_{H_{0}} \lesssim \inf _{\mathbf{A}_{h} \in H_{0 h}}\left\|\mathbf{B}-\mathbf{A}_{h}\right\|_{H_{0}}+\inf _{q_{h} \in Q_{0 h}}\left\|p-q_{h}\right\| \\
+\sup _{\mathbf{A}_{h} \in V_{h}} \frac{a\left(\mathbf{B}, \mathbf{A}_{h}\right)-a_{h}\left(\mathbf{B}, \mathbf{A}_{h}\right)}{\left\|\mathbf{A}_{h}\right\|_{H_{0 h}}} .
\end{gathered}
$$

Noting that for $\mathbf{B} \in H_{0}$, we have $S_{h} \mathbf{B} \in H_{0 h}$. On the other hand, for $p \in L_{0}^{2}(\Omega)$, let $\pi_{h} p$ be its standard $L^{2}$ projection in $Q_{h}$. Clearly $\pi_{h} p$ may not be in $Q_{0 h}$. But if we set $\tilde{p}_{h}=\pi_{h} p-\overline{\pi_{h} p}$, where $\bar{q}$ stands for the average of $q$ over $\Omega$ for any $q \in L^{2}(\Omega)$, then we have $\tilde{p}_{h} \in Q_{0 h}$, and the following estimates hold using the standard approximation property of the $L^{2}$ projection:

$$
\left\|p-\tilde{p}_{h}\right\|=\left\|\left(p-\pi_{h} p\right)-\overline{\left(p-\pi_{h} p\right)}\right\| \leq\left\|p-\pi_{h} p\right\| \lesssim h^{s_{2}}\|p\|_{s_{2}, \Omega} .
$$

Using this and Lemma 5.2, we derive by taking $\mathbf{A}_{h}=S_{h} \mathbf{B}$ and $q_{h}=\tilde{p}_{h}$ in (5.9) that

$$
\inf _{\mathbf{A}_{h} \in H_{0 h}}\left\|\mathbf{B}-\mathbf{A}_{h}\right\|_{H_{0}}+\inf _{q_{h} \in Q_{0 h}}\left\|p-q_{h}\right\| \lesssim h^{\frac{2 s_{1}}{3}} \sum_{i=1}^{3}\|\mathbf{B}\|_{1+s_{1}, \Omega_{i}}+h^{s_{2}}\|p\|_{s_{2}, \Omega}^{2} .
$$

It remains to estimate the last term in (5.9). Let $\mathcal{K}$ be the same as in Lemma 5.3, then by the definition of $a(\cdot, \cdot)$ and $a_{h}(\cdot, \cdot)$, we can write for any $\mathbf{A}_{h} \in H_{0 h}$ (cf. [10]),

$$
a\left(\mathbf{B}, \mathbf{A}_{h}\right)-a_{h}\left(\mathbf{B}, \mathbf{A}_{h}\right)=\sum_{K \in \mathcal{T}_{h}^{*}} \int_{\mathcal{K}}\left(\beta(x)-\beta_{h}(x)\right) \nabla \times \mathbf{B} \cdot \nabla \times \mathbf{A}_{h} d x .
$$

Using the Cauchy-Schwarz inequality, we obtain

$$
\begin{aligned}
& \left|a\left(\mathbf{B}, \mathbf{A}_{h}\right)-a_{h}\left(\mathbf{B}, \mathbf{A}_{h}\right)\right| \\
& \quad \lesssim \sum_{K \in \mathcal{T}_{h}^{*}}\left\{\left\|\nabla \times S_{h} \mathbf{B}\right\|_{0, \mathcal{K}}\left\|\nabla \times \mathbf{A}_{h}\right\|_{0, \mathcal{K}}+\left\|\nabla \times\left(\mathbf{B}-S_{h} \mathbf{B}\right)\right\|_{0, \mathcal{K}}\left\|\nabla \times \mathbf{A}_{h}\right\|_{0, \mathcal{K}}\right\} .
\end{aligned}
$$

By Lemmas 5.3 and 5.2, we deduce

$$
\begin{aligned}
\left|a\left(\mathbf{B}, \mathbf{A}_{h}\right)-a_{h}\left(\mathbf{B}, \mathbf{A}_{h}\right)\right| \lesssim & \sum_{K \in \mathcal{T}_{h}^{*}} h_{K}\left\|\nabla \times S_{h} \mathbf{B}\right\|_{0, K}\left\|\nabla \times \mathbf{A}_{h}\right\|_{0, K} \\
& +\sum_{K \in \mathcal{T}_{h}^{*}} h_{K}^{1 / 2}\left\|\nabla \times\left(\mathbf{B}-S_{h} \mathbf{B}\right)\right\|_{0, K}\left\|\nabla \times \mathbf{A}_{h}\right\|_{0, K} \\
\lesssim & h\left\|\nabla \times S_{h} \mathbf{B}\right\|_{0, \Omega}\left\|\nabla \times \mathbf{A}_{h}\right\|_{0, \Omega} \\
& +h^{1 / 2}\left\|\nabla \times\left(\mathbf{B}-S_{h} \mathbf{B}\right)\right\|_{0, \Omega}\left\|\nabla \times \mathbf{A}_{h}\right\|_{0, \Omega} \\
\lesssim & \left(h+h^{1 / 2+2 s_{1} / 3}\right)\left(\sum_{k=1}^{3}\|\mathbf{B}\|_{1+s_{1}, \Omega_{k}}\right)\left\|\nabla \times \mathbf{A}_{h}\right\|_{0, \Omega},
\end{aligned}
$$

and this completes the proof of Lemma 5.4.

Now, the above preparations enable us to make full use of the better local regularity of $\mathbf{B}$ in each subdomain $\Omega_{k}$ to derive the main results of this section, the finite element convergence. 
Theorem 5.1. Let $(\mathbf{B}, p) \in H^{2}\left(0, T ; H_{0}\right) \times L^{2}\left(0, T ; L_{0}^{2}(\Omega)\right)$ be the solution to the variational problem (3.7) such that $\mathbf{B} \in H^{1}\left(0, T ; H^{1+s_{1}}\left(\Omega_{k}\right)\right)$ in each $\Omega_{k}(k=1,2,3)$ for some $0 \leq s_{1}<1$ and $p \in H^{1}\left(0, T ; H^{s_{2}}(\Omega)\right)$ for some $0 \leq s_{2}<1$. And let $\left(\mathbf{B}_{h}, p_{h}\right)$ be the finite element solution to the fully discrete finite element approximation (4.15), then we have the following error estimates:

$$
\begin{aligned}
& \max _{1 \leq n \leq M}\left\|\mathbf{B}_{h}^{n}-\mathbf{B}^{n}\right\|^{2}+\tau \sum_{n=1}^{M}\left\{\left\|\nabla \times\left(\mathbf{B}_{h}^{n}-\mathbf{B}^{n}\right)\right\|^{2}+\left\|\nabla \cdot\left(\mathbf{B}_{h}^{n}-\mathbf{B}^{n}\right)\right\|^{2}\right\} \\
\lesssim & h^{\frac{4 s_{1}}{3}} \sum_{k=1}^{3}\|\mathbf{B}\|_{H^{1}\left(0, T ; H^{1+s_{1}}\left(\Omega_{k}\right)\right)}^{2}+h^{2 s_{2}}\|p\|_{H^{1}\left(0, T ; H^{s_{2}}(\Omega)\right)}^{2} \\
& +\tau^{2}\left\{\|\mathbf{B}\|_{H^{2}\left(0, T ; H_{0}\right)}^{2}+\|p\|_{L^{2}\left(Q_{T}\right)}^{2}\right\} .
\end{aligned}
$$

Proof. Our aim is to estimate the error $\left(\mathbf{B}^{n}-\mathbf{B}_{h}^{n}\right)$. Using the relation

$$
\mathbf{B}^{n}-\mathbf{B}_{h}^{n}=\left(\mathbf{B}^{n}-\overline{\mathbf{B}}^{n}\right)+\left(\overline{\mathbf{B}}^{n}-P_{h} \overline{\mathbf{B}}^{n}\right)+\left(P_{h} \overline{\mathbf{B}}^{n}-\mathbf{B}_{h}^{n}\right),
$$

and the projection results from Lemma 5.4, it suffices to estimate the difference $\xi_{h}^{n}=$ $\left(P_{h} \overline{\mathbf{B}}^{n}-\mathbf{B}_{h}^{n}\right)$ in the specified norms. To do so, letting $\mathbf{A}=\tau^{-1} \mathbf{A}_{h} \in H_{0 h}$ and $q=q_{h} \in Q_{0 h}$ in (3.7), then integrating over $\left[t_{n-1}, t_{n}\right]$ we obtain

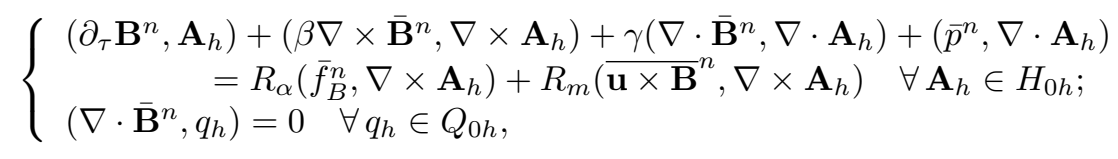

where

$$
\bar{f}_{B}^{n}=\frac{1}{\tau} \int_{t_{n-1}}^{t_{n}} \frac{f}{1+\sigma|\mathbf{B}|^{2}} \mathbf{B}(t) d t .
$$

Subtracting (4.15) from (5.12) yields

$$
\begin{aligned}
& \left(\partial_{\tau} \xi_{h}^{n}, \mathbf{A}_{h}\right)+\left(\beta_{h} \nabla \times \xi_{h}^{n}, \nabla \times \mathbf{A}_{h}\right)+\gamma\left(\nabla \cdot \xi_{h}^{n}, \nabla \cdot \mathbf{A}_{h}\right) \\
& =\left(\partial_{\tau}\left(P_{h} \overline{\mathbf{B}}^{n}-\mathbf{B}^{n}\right), \mathbf{A}_{h}\right)+R_{\alpha}\left(\bar{f}_{B}^{n}-\frac{f_{h}^{n}}{1+\sigma\left|\mathbf{B}_{h}^{n-1}\right|^{2}} \mathbf{B}_{h}^{n}, \nabla \times \mathbf{A}_{h}\right), \\
& +R_{m}\left({\overline{\mathbf{u} \times \mathbf{B}^{n}}}^{n}-\mathbf{u}_{h}^{n} \times \mathbf{B}_{h}^{n}, \nabla \times \mathbf{A}_{h}\right) \\
& +\left(\beta_{h} \nabla \times P_{h} \overline{\mathbf{B}}^{n}-\beta \nabla \times \overline{\mathbf{B}}^{n}, \nabla \times \mathbf{A}_{h}\right)+\left(\nabla \cdot\left(P_{h} \overline{\mathbf{B}}^{n}-\overline{\mathbf{B}}^{n}\right), \nabla \cdot \mathbf{A}_{h}\right) \\
& +\left(P_{h} \bar{p}^{n}-\bar{p}^{n}, \nabla \cdot \mathbf{A}_{h}\right)+\left(p_{h}^{n}-P_{h} \bar{p}^{n}, \nabla \cdot \mathbf{A}_{h}\right) \quad \forall \mathbf{A}_{h} \in H_{0 h} .
\end{aligned}
$$

Letting $\mathbf{A}_{h}=\tau \xi_{h}^{n} \in H_{0 h}$ above, then using the second equations in both (4.15) and (5.12) and the definition of the projection $P_{h}$, we come to (cf. [10])

$$
\begin{aligned}
& \tau\left(\partial_{\tau} \xi_{h}^{n}, \xi_{h}^{n}\right)+\tau\left(\beta_{h} \nabla \times \xi_{h}^{n}, \nabla \times \xi_{h}^{n}\right)+\gamma \tau\left(\nabla \cdot \xi_{h}^{n}, \nabla \cdot \xi_{h}^{n}\right) \\
& =R_{\alpha} \tau\left(\bar{f}_{B}^{n}-\frac{f^{n}}{1+\sigma\left|\mathbf{B}_{h}^{n-1}\right|^{2}} \mathbf{B}_{h}^{n}, \nabla \times \xi_{h}^{n}\right)+R_{m} \tau\left({\overline{\mathbf{u} \times \mathbf{B}^{n}}}^{n} \mathbf{u}^{n} \times \mathbf{B}_{h}^{n}, \nabla \times \xi_{h}^{n}\right) \\
& +\tau\left(\partial_{\tau}\left(P_{h} \overline{\mathbf{B}}^{n}-\mathbf{B}^{n}\right), \xi_{h}^{n}\right)+\tau\left(\overline{\mathbf{B}}^{n}-P_{h} \overline{\mathbf{B}}^{n}, \xi_{h}^{n}\right) \\
& +\tau\left(\frac{R_{\alpha}\left(f^{n}-f_{h}^{n}\right)}{1+\sigma\left|\mathbf{B}_{h}^{n-1}\right|^{2}} \mathbf{B}_{h}^{n}+R_{m}\left(\mathbf{u}^{n}-\mathbf{u}_{h}^{n}\right) \times \mathbf{B}_{h}^{n}, \nabla \times \xi_{h}^{n}\right) \\
& (5.13) \equiv:(\mathrm{I})_{1}+(\mathrm{I})_{2}+(\mathrm{I})_{3}+(\mathrm{I})_{4}+(\mathrm{I})_{5} \text {. }
\end{aligned}
$$


Obviously, $(\mathrm{I})_{4}$ can be estimated immediately by the projection property. Below, we shall analyze $(\mathrm{I})_{1},(\mathrm{I})_{2},(\mathrm{I})_{3}$, and $(\mathrm{I})_{5}$ one by one. For the estimation of $(\mathrm{I})_{1}$, we first consider

$$
(\mathrm{II})_{1} \equiv: \bar{f}_{B}^{n}-\frac{f^{n}}{1+\sigma\left|\mathbf{B}_{h}^{n-1}\right|^{2}} \mathbf{B}_{h}^{n} .
$$

By direct manipulations, we have (cf. [10])

$$
\begin{aligned}
(\mathrm{II})_{1}= & \frac{1}{\tau} \int_{t_{n-1}}^{t_{n}} \frac{\left(f-f^{n}\right)+\sigma f\left(\left|\mathbf{B}_{h}^{n-1}\right|^{2}-|\mathbf{B}|^{2}\right)+\sigma\left(f-f^{n}\right)|\mathbf{B}|^{2}}{\left(1+\sigma|\mathbf{B}|^{2}\right)\left(1+\sigma\left|\mathbf{B}_{h}^{n-1}\right|^{2}\right)} \mathbf{B}(t) d t \\
& +\frac{1}{\tau} \int_{t_{n-1}}^{t_{n}} \frac{f^{n}}{1+\sigma\left|\mathbf{B}_{h}^{n-1}\right|^{2}}\left\{\left(\mathbf{B}(t)-\overline{\mathbf{B}}^{n}\right)+\left(\overline{\mathbf{B}}^{n}-P_{h} \overline{\mathbf{B}}^{n}\right)+\left(P_{h} \overline{\mathbf{B}}^{n}-\mathbf{B}_{h}^{n}\right)\right\} d t,
\end{aligned}
$$

which can be easily bounded by

$$
\begin{aligned}
\left|(\mathrm{II})_{1}\right| \leq & \frac{2}{\tau} \int_{t_{n-1}}^{t_{n}}|f|\left\{\left|\mathbf{B}_{h}^{n-1}-P_{h} \overline{\mathbf{B}}^{n-1}\right|+\left|P_{h} \overline{\mathbf{B}}^{n-1}-\overline{\mathbf{B}}^{n-1}\right|+\left|\overline{\mathbf{B}}^{n-1}-\mathbf{B}\right|\right\} d t \\
& +\frac{1}{\tau} \int_{t_{n-1}}^{t_{n}}\left|f-f^{n}\right||\mathbf{B}(t)| d t+\frac{1}{\tau} \int_{t_{n-1}}^{t_{n}}\left|f^{n}\right|\left(\left|\mathbf{B}(t)-\overline{\mathbf{B}}^{n}\right|+\left|\overline{\mathbf{B}}^{n}-P_{h} \overline{\mathbf{B}}^{n}\right|+\left|\xi_{h}^{n}\right|\right) d t .
\end{aligned}
$$

By the standard error estimates $[5,14,10]$, we obtain from (5.14) that

$$
\left|(\mathrm{II})_{1}\right| \leq 5 f_{M}\left\{\sqrt{\tau}\|\mathbf{B}\|_{L^{2}\left(t_{n-1}, t_{n}\right)}+\sqrt{\tau}\left\|\mathbf{B}_{t}\right\|_{L^{2}\left(t_{n-2}, t_{n}\right)}+\sum_{k=n-1}^{n}\left|\xi_{h}^{k}\right|+\sum_{k=n-1}^{n}\left|P_{h} \overline{\mathbf{B}}^{k}-\overline{\mathbf{B}}^{k}\right|\right\} .
$$

Similarly, for the estimation of (I) $)_{2}$, we first analyze $\left(\mathrm{II}_{2}\right):={\overline{\mathbf{u} \times \mathbf{B}^{n}}}^{n} \mathbf{u}^{n} \times \mathbf{B}_{h}^{n}$. We write

$$
(\mathrm{II})_{2}=\frac{1}{\tau} \int_{t_{n-1}}^{t_{n}}\left\{\left(\mathbf{u}(t)-\mathbf{u}^{n}\right) \times \mathbf{B}(t)+\mathbf{u}^{n} \times\left(\mathbf{B}(t)-\mathbf{B}_{h}^{n}\right)\right\} d t,
$$

this leads readily to (with $\left.I_{n}=\left(t_{n-1}, t_{n}\right]\right)$

$$
\left|(\mathrm{II})_{2}\right| \leq u_{M} \sqrt{\tau}\|\mathbf{B}\|_{L^{2}\left(I_{n}\right)}+2 u_{M}\left(\sqrt{\tau}\left\|\mathbf{B}_{t}\right\|_{L^{2}\left(I_{n}\right)}+\left|\overline{\mathbf{B}}^{n}-P_{h} \overline{\mathbf{B}}^{n}\right|+\left|\xi_{h}^{n}\right|\right) .
$$

Next, we estimate the following term needed in (5.13):

$$
\begin{aligned}
(\mathrm{II})_{3} & \equiv: \partial_{\tau}\left(P_{h} \overline{\mathbf{B}}^{n}-\mathbf{B}^{n}\right)=\partial_{\tau}\left(P_{h} \mathbf{B}^{n}-\mathbf{B}^{n}\right)+P_{h} \partial_{\tau}\left(\overline{\mathbf{B}}^{n}-\mathbf{B}^{n}\right) \\
& =\frac{1}{\tau} \int_{t_{n-1}}^{t_{n}}\left(P_{h} \mathbf{B}_{t}(s)-\mathbf{B}_{t}(s)\right) d s+P_{h} \partial_{\tau}\left(\overline{\mathbf{B}}^{n}-\mathbf{B}^{n}\right) .
\end{aligned}
$$

For the second term above, we can write after some manipulations [10] that

$$
\partial_{\tau}\left(\overline{\mathbf{B}}^{n}-\mathbf{B}^{n}\right)=\frac{1}{\tau^{2}} \int_{t_{n-1}}^{t_{n}} \int_{s-\tau}^{s} \int_{s}^{\mu} \mathbf{B}_{t t}(\lambda) d \lambda d \mu d s,
$$

this enables us to rewrite $(\mathrm{II})_{3}$ as

$$
(\mathrm{II})_{3}=\frac{1}{\tau} \int_{t_{n-1}}^{t_{n}}\left(P_{h} \mathbf{B}_{t}(s)-\mathbf{B}_{t}(s)\right) d s+\frac{1}{\tau^{2}} \int_{t_{n-1}}^{t_{n}} \int_{s-\tau}^{s} \int_{s}^{\mu} P_{h} \mathbf{B}_{t t}(\lambda) d \lambda d \mu d s,
$$


and so,

$$
\left|(\mathrm{II})_{3}\right| \leq \frac{1}{\sqrt{\tau}}\left\|P_{h} \mathbf{B}_{t}-\mathbf{B}_{t}\right\|_{L^{2}\left(I_{n}\right)}+\sqrt{\tau}\left\|P_{h} \mathbf{B}_{t t}\right\|_{L^{2}\left(I_{n}\right)} .
$$

For the last term $(\mathrm{I})_{5}$ in (5.13), by Lemma 5.3 and Young's inequality there exists some constant $\tilde{C}$ independent of $\tau$ and $h$ such that [10]

$$
\begin{aligned}
\left|(\mathrm{I})_{5}\right| & \leq 2 \tau h \tilde{C}\left(R_{\alpha} f_{M}+2 R_{m} u_{M}\right) \sum_{K \in \mathcal{T}_{h}^{*}}\left\|\mathbf{B}_{h}^{n}\right\|_{0, K}\left\|\nabla \times \xi_{h}^{n}\right\|_{0, K} \\
& \leq \frac{\beta_{m}}{4} \tau\left\|\nabla \times \xi_{h}^{n}\right\|^{2}+C \tau h^{2}\left\|\mathbf{B}_{h}^{n}\right\|^{2} .
\end{aligned}
$$

Now, we obtain from (5.13) and (5.19) and Young's inequality that

$$
\left\|\xi_{h}^{n}\right\|^{2}-\left\|\xi_{h}^{n-1}\right\|^{2}+\tau \beta_{m}\left\|\nabla \times \xi_{h}^{n}\right\|^{2}+\gamma \tau\left\|\nabla \cdot \xi_{h}^{n}\right\|^{2} \lesssim(\mathrm{III})_{1},
$$

where the term $(\mathrm{III})_{1}$ is given by

$$
(\mathrm{III})_{1}=\tau\left\{\left\|(\mathrm{II})_{1}\right\|^{2}+\left\|(\mathrm{II})_{2}\right\|^{2}+\left\|(\mathrm{II})_{3}\right\|^{2}+\left\|\overline{\mathbf{B}}^{n}-P_{h} \overline{\mathbf{B}}^{n}\right\|^{2}+\left\|\xi_{h}^{n}\right\|^{2}+h^{2}\left\|\mathbf{B}_{h}^{n}\right\|^{2}\right\} .
$$

Using the estimates for $(\mathrm{II})_{1},(\mathrm{II})_{2}$, and $(\mathrm{II})_{3}$ in (5.15)-(5.18), we can further estimate (III) $)_{1}$ by

$$
\begin{aligned}
(\mathrm{III})_{1} \lesssim & \int_{t_{n-1}}^{t_{n}}\left\|P_{h} \mathbf{B}_{t}(s)-\mathbf{B}_{t}(s)\right\|^{2} d s+\tau^{2} \int_{t_{n-1}}^{t_{n}}\left\|P_{h} \mathbf{B}_{t t}(t)\right\|^{2} d t+\tau\left\{\left\|\xi_{h}^{n-1}\right\|^{2}+\left\|\xi_{h}^{n}\right\|^{2}\right\} \\
& +\tau\left\{\sum_{k=n-1}^{n}\left\|P_{h} \overline{\mathbf{B}}^{k}-\overline{\mathbf{B}}^{k}\right\|^{2}+\tau \int_{t_{n-2}}^{t_{n}}\left(\|\mathbf{B}(t)\|^{2}+\left\|\mathbf{B}_{t}(t)\right\|^{2}\right) d t\right\}+\tau h^{2}\left\|\mathbf{B}_{h}^{n}\right\|^{2} .
\end{aligned}
$$

Summing both sides of (5.20) over $n=1,2, \ldots, k \leq M$ yields

$$
\begin{aligned}
& \left\|\xi_{h}^{k}\right\|^{2}+\beta_{m} \tau \sum_{n=1}^{k}\left\|\nabla \times \xi_{h}^{n}\right\|^{2}+2 \tau \sum_{n=1}^{k}\left\|\nabla \cdot \xi_{h}^{n}\right\|^{2} \\
\lesssim & \left\|\xi_{h}^{0}\right\|^{2}+\left\|P_{h} \mathbf{B}(0)-\mathbf{B}(0)\right\|^{2}+\tau \sum_{n=1}^{k}\left\|\xi_{h}^{n}\right\|^{2}+\tau h^{2} \sum_{n=1}^{k}\left\|\mathbf{B}_{h}^{n}\right\|^{2} \\
& +\int_{0}^{T}\left\|P_{h} \mathbf{B}_{t}(t)-\mathbf{B}_{t}(t)\right\|^{2} d t+\tau^{2} \int_{0}^{T}\left\|P_{h} \mathbf{B}_{t t}(t)\right\|^{2} d t \\
& \left.+\tau \sum_{n=1}^{k}\left\|P_{h} \overline{\mathbf{B}}^{n}-\overline{\mathbf{B}}^{n}\right\|^{2}+\tau \int_{0}^{T}\left(\|\mathbf{B}(t)\|^{2}+\left\|\mathbf{B}_{t}(t)\right\|^{2}\right) d t\right\} .
\end{aligned}
$$

Finally using Lemmas 5.1, 5.2, and 5.4, and applying the discrete Gronwall inequality, we are led to the error estimates in Theorem 5.1.

6. Application to a solar interface dynamo. For the astrophysical application of the mathematical theory, we shall concentrate on the numerical modelling of solar interface dynamos. Helioseismology reveals the existence of a highly differentially rotating transition zone at the bottom of the convection zone, which is usually referred to as the solar tachocline [28]. It is thought that the tachocline offers an ideal location for the generation and storage of the Sun's strong toroidal magnetic fields. 
In other words, the large-scale solar surface magnetic activities can be interpreted as a result of the rising and emerging of tachocline-seated, strong toroidal magnetic fields driven by magnetic buoyancy [34]. The existence of the tachocline leads to development of the solar interface dynamo first proposed by Parker [25], in which the generation of a weak poloidal magnetic field and a strong toroidal magnetic field takes place in separate fluid regions. Parker's interface dynamo concept depicts an attractive picture of generating a strong toroidal magnetic field within the tachocline while avoiding the dilemma relating to the strong alpha quenching in the convection zone. We shall apply the finite element dynamo theory and algorithm discussed in the previous sections to the problem of solar interface dynamo modelling.

In the solar interface dynamo model, we shall take $\mathbf{u}$ as the solar-like internal differential rotation profile, a result of the helioseismic inversion (e.g., [28]) while the function $f$ is assumed to be given by

$$
f(\mathbf{x}, t)=\sin ^{2} \theta \cos \theta \sin \left[\pi \frac{\left(r-r_{1}\right)}{\left(r_{2}-r_{1}\right)}\right],
$$

where $(r, \theta, \phi)$ is the spherical polar coordinates. Similar forms have been used in the previous solar dynamo simulations $[27,21,16]$. Furthermore, the weaker pole-equator differential rotation in the convection zone is neglected and the amplification of the toroidal magnetic field only occurs in the tachocline. It follows that the two magnetic induction sources, the generation of a poloidal field in the convection zone, and the amplification of the toroidal field in the tachocline, is spatially separated, as suggested by Parker [25].

We have simulated three nonlinear finite element dynamos at $R_{\alpha}=30$ for different magnetic Reynolds numbers, $R_{m}=100,200$, and 500. Figure 2 displays magnetic energies of the three nonlinear dynamo solutions as a function of time. The corresponding butterfly diagram, contours of the azimuthal magnetic field evaluated at the bottom of the convection zone plotted against time, is also shown in Figure 2 for the case with $R_{m}=200$. In Figure 3, we illustrate the time-dependent spatial structure of the generated magnetic field in a meridional plane for $R_{m}=200$, showing an equatorially propagating dynamo wave similar to that of the solar cycle.
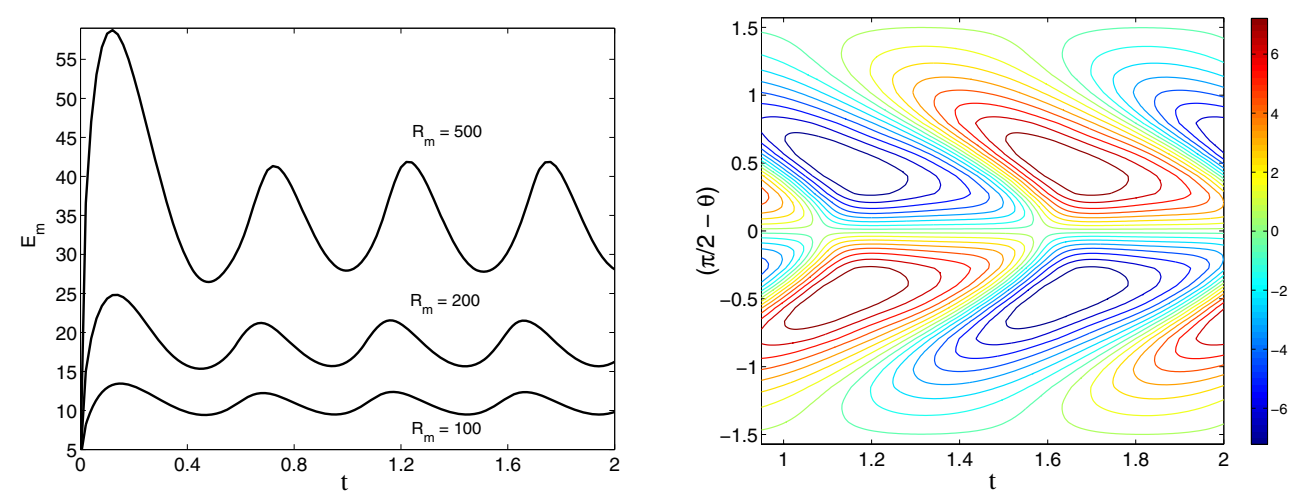

FIG. 2. The left panel shows magnetic energy $E_{m}$ of the dynamo as a function of time with a steady tachocline for different values of $R_{m}$ at $R_{\alpha}=30$ with $\beta_{1}=1, \beta_{2}=1$, and $\beta_{3}=150$. The right panel displays "a butterfly diagram" for the solution $R_{m}=200$ with the azimuthal magnetic field evaluated at the bottom of the convection zone. 

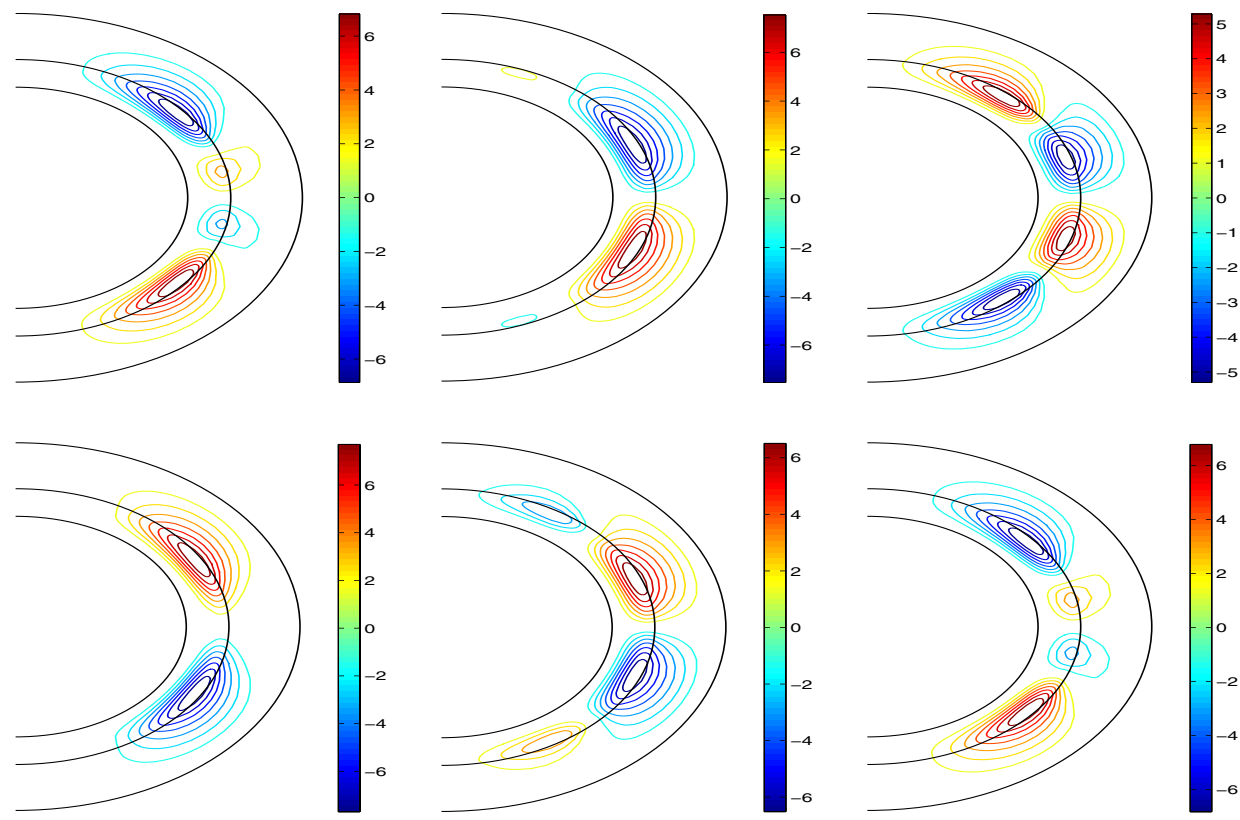

FIG. 3. Contours of the azimuthal field $B_{\phi}$ in a meridional plane plotted at six different instants for $t=1.0,1.2,1.4,1.6,1.8,2.0$ (from top left to right and then from lower left to right) for $R_{m}=200$, $\beta_{1}=1, \beta_{2}=1$, and $\beta_{3}=150$.

There are a number of important features shown in our finite element dynamo solutions. First, the effect of the large-scale differential rotation $\mathbf{u}$ in the tachocline always gives rise to an oscillatory dynamo with a period of about one magnetic diffusion unit, which is about 10 years if we adopt $\lambda_{2}=10^{8} \mathrm{~m}^{2} \mathrm{~s}^{-1}$, approximate to what has been observed in the solar magnetic field. Second, the interface dynamo solutions always select dipolar symmetry and propagate equator ward though the numerical simulation is fully three-dimensional, which is again consistent with the observed feature of the solar magnetic field. Finally, the generated magnetic field mainly concentrates in the vicinity of the interface between the tachocline and the convection zone. A strong toroidal magnetic field in the tachocline is likely to be susceptible to magnetic buoyancy instabilities leading to a quick eruption of the field into the surface of the Sun in the form of sunspots.

7. Concluding remarks. Modelling stellar and planetary dynamos represents an important, highly active research front in astrophysics and planetary physics. Nearly all current stellar dynamo models are based on spectral methods in terms of spherical harmonic expansions, which are computationally inefficient on modern parallel computers and limit the application to general dynamo models, especially to the models with variable physical coefficients of space and time. The finite element method discussed in this paper offers an attractive alternative for simulating dynamos in spherical geometry.

The first attempt at using finite element methods for numerical simulations of spherical dynamos was made in [11]. The current work presents the first mathematical theory and numerical analysis for mean-field spherical dynamos, and it has made contributions in the following aspects: (1) The well-posedness of the mean-field 
dynamo system is rigorously demonstrated; the dynamo system is characterized in terms of a saddle-point type formulation which can be conveniently approximated by finite element methods. (2) The existing convergence theory on saddle-point systems is improved and generalized so that the symmetric part of the bilinear form allows the approximations of curved interfaces by straight polygons and numerical integrations on polyhedra with curved faces are replaced by much easier integrations on polyhedra with planar faces; and this is the first work of such type on saddle-point systems. (3) A fully discrete finite element method is proposed for the interface dynamo system with discontinuous coefficients, and error estimates are established under very weak global and local regularity assumptions on the solutions, and this work seems to be the first in achieving error estimates of numerical methods for three-dimensional interface PDEs with curved interfaces, especially for nonlinear PDEs. (4) The application of the proposed numerical method to a solar interface dynamo verifies some important physical observations.

We believe that the mathematical theory and finite element methods for spherical dynamos and their successful application to the solar interface dynamo presented in this paper open up an exciting opportunity for future numerical simulation of stellar dynamos. We also believe that the finite element theory and method developed in the paper would also benefit other research communities in geophysics, planetary physics, and astrophysics where the magnetic field and spherical geometry play an essential role.

\section{REFERENCES}

[1] M. H. Acuna And N. F. Ness, Jupiter's main magnetic field measured by Pioneer 11, Nature, 253 (1975), pp. 327-328.

[2] F. Assous, P. Degond, E. Heintzé, P. A. Raviart, and J. Segré, On a finite element method for solving the three-dimensional Maxwell equations, J. Comput. Phys., 109 (1993), pp. 222-237.

[3] A. Brandenburg, The case for a distributed solar dynamo shaped by near-surface shear, Astrophy. J., 625 (2005), pp. 539-547.

[4] A. Brandenburg and K. Subramanian, Astrophyiscal magnetic fields and nonlinear dynamo theory, Physics Reports, 417 (2005), pp. 1-209.

[5] H. T. BANKS AND J. ZOU, Regularity and approximation of systems arising in electromagnetic interrogation of dielectric material, Numer. Funct. Anal. Optim., 20 (1999), pp. 609-627.

[6] F. Brezzi And M. Fortin, Mixed and Hybrid Finite Element Methods, Springer-Verlag, New York, 1991.

[7] A. S. BRUn AND J. ToOmRe, Turbulent convection under the influence of rotation: sustaining a strong differential rotation, Astrophy. J., 570 (2002), pp. 865-885.

[8] B. F. BuRKE AND K. L. FrankLin, Observations of a variable radio source associated with the planet Jupiter, J. Geophys. Res., 60 (1952), pp. 213-217.

[9] F. Cattaneo and D. W. Hughes, Nonlinear saturation of the turbulent alpha effect where a large scale field is imposed, Phys. Rev. E, 54 (1996), pp. R4532-R4535.

[10] K. H. Chan, K. Zhang, and J. Zou, Spherical Interface Dynamos: Mathematical Theory, Finite Element Approximation and Application, Technical Report CUHK-2005-10 (331), Department of Mathematics, The Chinese University of Hong Kong, Hong Kong, 2005, available online at http://www.math.cuhk.edu.hk/en/report/index.php.

[11] K. H. Chan, K. Zhang, J. Zou, And G. Schubert, A nonlinear 3-D spherical alpha-square dynamo using a finite element method, Phys. Earth Planet. Int., 128 (2001), pp. 35-50.

[12] Z. Chen, Q. Du, AND J. Zou, Finite element methods with matching and nonmatching meshes for Maxwell equations with discontinuous coefficients, SIAM J. Numer. Anal., 37 (2000), pp. $1542-1570$.

[13] P. Ciarlet, Jr., and J. Zou, Finite element convergence for the Darwin model to Maxwell's equations, RAIRO Math. Model. Numer. Anal., 31 (1997), pp. 213-249.

[14] P. Ciarlet, Jr., AND J. Zou, Fully discrete finite element approaches for time-dependent Maxwell's equations, Numer. Math., 82 (1999), pp. 193-219. 
[15] P. G. Ciarlet, The Finite Element Method for Elliptic Problems, North-Holland Publishing, Amsterdam-New York-Oxford, 1978.

[16] M. Dikpati and P. Charbonneau, A Babcock-Leighton flux transport dynamo with solar-like differential rotation, Astrophy. J., 518 (1999), pp. 508-520.

[17] M. Feistauer and A. Zenisek, Finite element solution of nonlinear elliptic problems, Numer. Math., 50 (1987), pp. 451-475.

[18] V. Girault and P. Raviart, Finite Element Methods for Navier-Stokes Equations, SpringerVerlag, Berlin, 1986.

[19] G. Glatzmaier and P. Roberts, A three-dimensional convective dynamo solution with rotating and finitely conducting inner core and mantle, Phys. Earth Planet. Int., 91 (1995), pp. $63-75$.

[20] P. Hood AND G. TAYLOR, Navier-Stokes equations using mixed interpolation, in Finite Element Methods in Flow Problems, J. Oden, ed., UAH Press, Huntsville, AL, 1974.

[21] J. A. Markiel and J. H. Thomas, Solar interface dynamo models with a realistic rotation profile, Astrophy. J., 523 (1999), pp. 827-837.

[22] M. S. Miesch, J. R. Elliott, J. Toomre, T. L. Clune, G. A. Glatzmaier, and P. A. Gilman, Three-dimensional spherical simulations of solar convection. I. Differential rotation and pattern evolution achieved with laminar and turbulent states, Astrophy. J., 532 (2000), pp. 593-615.

[23] H. K. Moffatt, Magnetic Field Generation in Electrically Conducting Fluids, Cambridge University Press, Cambridge, UK, 1978.

[24] E. N. Parker, Cosmical Magnetic Fields, Clarendon Press, Oxford, 1979.

[25] E. N. PARKer, A solar dynamo surface wave at the interface between convection and nonuniform rotation, Astrophy. J., 408 (1993), pp. 707-719.

[26] P. H. Roberts And A. M. Soward, Dynamo theory, in Annual Review of Fluid Mechanics, Vol. 24, Annual Reviews, Palo Alto, CA, 1992, pp. 459-512.

[27] G. Ruediger and A. Brandenburg, A solar dynamo in the overshoot layer: Cycle period and butterfly diagram, Astronomy and Astrophysics, 296 (1995), pp. 557-566.

[28] J. SchOU ET AL., Helioseismic studies of differential rotation in the solar envelope by the solar oscillations investigation using Michelson Doppler Imager, Astrophy. J, 505 (1998), pp. 390-417.

[29] L. R. Scott and S. Zhang, Finite element interpolation of nonsmooth functions satisfying boundary conditions, Math. Comp., 54 (1990), pp. 483-493.

[30] E. M. Stein, Singular Integrals and Differentiability Properties of Functions, Princeton University Press, Princeton, NJ, 1970.

[31] M. Stix, The Sun: An Introduction, Springer-Verlag, Berlin, 2002.

[32] R. Temam, Navier-Stokes Equations: Theory and Numerical Analysis, North-Holland Publishing, Amsterdam, 1977.

[33] R. VerfürTh, Error estimates for a mixed finite element approximation of the Stokes equations, RAIRO Model. Math. Numer. Anal., 18 (1984), pp. 175-182.

[34] N. O. Weiss, in Lectures on Solar and Planetary Dynamos, M. R. E. Proctor and A. D. Gilbert, eds., Cambridge University Press, England, 1994.

[35] K. Zhang AND F. Busse, Convection driven magnetohydrodynamic dynamos in rotating spherical shells, Geophys. Astrophys. Fluid Dyn., 49 (1989), pp. 97-116.

[36] K. Zhang And G. SChUbert, Magnetohydrodynamics in rapidly rotating spherical systems, in Annual Review of Fluid Mechanics, Vol. 32, Annual Reviews, Palo Alto, CA, 2000, pp. 411-445. 